\title{
Melnikov theory to all orders and Puiseux series for subharmonic solutions
}

\author{
Livia Corsi and Guido Gentile \\ Dipartimento di Matematica, Università di Roma Tre, Roma, I-00146, Italy. \\ E-mail: lcorsi@mat.uniroma3.it, gentile@mat.uniroma3.it
}

\begin{abstract}
We study the problem of subharmonic bifurcations for analytic systems in the plane with perturbations depending periodically on time, in the case in which we only assume that the subharmonic Melnikov function has at least one zero. If the order of zero is odd, then there is always at least one subharmonic solution, whereas if the order is even in general other conditions have to be assumed to guarantee the existence of subharmonic solutions. Even when such solutions exist, in general they are not analytic in the perturbation parameter. We show that they are analytic in a fractional power of the perturbation parameter. To obtain a fully constructive algorithm which allows us not only to prove existence but also to obtain bounds on the radius of analyticity and to approximate the solutions within any fixed accuracy, we need further assumptions. The method we use to construct the solution - when this is possible - is based on a combination of the Newton-Puiseux algorithm and the tree formalism. This leads to a graphical representation of the solution in terms of diagrams. Finally, if the subharmonic Melnikov function is identically zero, we show that it is possible to introduce higher order generalisations, for which the same kind of analysis can be carried out.
\end{abstract}

\section{Introduction}

The problem of subharmonic bifurcations was first considered by Melnikov [8, who showed that the existence of subharmonic solutions is related to the zeroes of a suitable function, nowadays called the subharmonic Melnikov function. The standard Melnikov theory usually studies the case in which the subharmonic Melnikov function has a simple (i.e. first order) zero [3, 7]. In such a case the problem can be reduced to a problem of implicit function theorem.

Nonetheless, it can happen that the subharmonic Melnikov function either vanishes identically or has a zero which is of order higher than one. In the first case hopefully one can go to higher orders, and if a suitable higher order generalisation of the subharmonic Melnikov function has a first order zero, then one can proceed very closely to the standard case, and existence of analytic subharmonic solutions is obtained. Most of the papers in the literature consider this kind of generalisations of Melnikov's theory, and often a second order analysis is enough to settle the problem.

The second case is more subtle. The problem can be still reduced to an implicit function problem, but the fact that the zeroes are no longer simple prevents us from applying the implicit function theorem. Thus, other arguments must be used, based on the Weierstrass preparation theorem and on the theory of the Puiseux series [11, 2, 3, 1. However, a systematic analysis is missing in the literature. Furthermore, in general, these arguments are not constructive: if on the one hand they allow to prove (in certain cases) the existence of at least one subharmonic solution, on the other hand the problem of how many such solutions really exist and how they can be explicitly constructed has not been discussed in full generality. 
The main difficulty for a constructive approach is that the solution of the implicit function equation has to be looked for by successive approximations. At each iteration step, in order to find the correction to the approximate solution found at the previous one, one has to solve a new implicit function equation, which, in principle, still admits multiple roots. So, as far as the roots of the equations are not simple, one cannot give an algorithm to produce systematically the corrections at the subsequent steps.

A careful discussion of a problem of the same kind can be found in [1, where the problem of bifurcations from multiple limit cycles is considered - cf. also [9, 10, where the problem is further investigated. There, under the hypothesis that a simple (real) zero is obtained at the first iteration step, it is proved that the bifurcating solutions can be expanded as fractional series (Puiseux series) of the perturbation parameter. The method to compute the coefficients of the series is based on the use of Newton's polygon 2, 3, 1, and allows one to go to arbitrarily high orders. However, the convergence of the series, and hence of the algorithm, relies on abstract arguments of algebraic and geometric theory.

To the best of our knowledge, the case of subharmonic bifurcations was not discussed in the literature. Of course, in principle one can think to adapt the same strategy as in [1] for the bifurcations of limit cycles. But still, there are issues which have not been discussed there. Moreover we have a twofold aim. We are interested in results which are both general - not generic - and constructive. This means that we are interested in problems such as the following one: which are the weaker conditions to impose on the perturbation, for a given integrable system and a given periodic solution, in order to prove the existence of subharmonic solutions? Of course the ideal result would be to have no restriction at all. At the same time, we are also interested in explicitly construct such solutions, within any prefixed accuracy.

The problem of subharmonic solutions in the case of multiple zeroes of the Melnikov functions has been considered in [14, where the following theorem is stated (without giving the proof) for $C^{r}$ smooth systems: if the subharmonic Melnikov function has a zero of order $n \leq r$, then there is at least one subharmonic solution. In any case the analyticity properties of the solutions are not discussed. In particular the subharmonic solution is found as a function of two parameters - the perturbation parameter and the initial phase of the solution to be continued -, but the relation between the two parameters is not discussed. We note that, in the analytic setting, it is exactly this relation which produces the lack of analyticity in the perturbation parameter. Furthermore, in [14 the case of zeroes of even order is not considered: as we shall see, in that case the existence of subharmonic solutions can not be proved in general, but it can be obtained under extra assumptions.

In the remaining part of this section, we give a more detailed account of our results. One can formulate the problem both in the $C^{r}$ Whitney topology and in the real-analytic setting. We shall choose the latter. From a technical point of view, this is mandatory since our techniques requires for the systems to be analytic. However, it is also very natural from a physical point of view, because in practice in any physical applications the functions appearing in the equations are analytic (often even polynomials), and when they are not analytic they are not even smooth. Also, we note since now that, even though we restrict our analysis to the analytic setting, this does not mean at all that we can not deal with problems where non-analytic phenomena arise. The very case discussed in this paper provides a counterexample.

We shall consider systems which can be viewed as perturbations of integrable systems, with the perturbation which depends periodically in time. We shall use coordinates $(\alpha, A)$ such that, in the absence of the perturbation, $A$ is fixed to a constant value, while $\alpha$ rotates on the circle: hence all motions are periodic. As usual [7] we assume that, for $A$ varying in a finite interval, the periods change monotonically. Then we can write the equations of motion as $\dot{\alpha}=\omega(A)+\varepsilon F(\alpha, A, t), \dot{A}=\varepsilon G(\alpha, A, t)$, with $G, F$ periodic in $\alpha$ and $t$. All functions are assumed to be analytic. More formal definitions will be given in Section 2 ,

Given a unperturbed periodic orbit $t \rightarrow\left(\alpha_{0}(t), A_{0}(t)\right)$, we define the subharmonic Melnikov function $M\left(t_{0}\right)$ as the average over a period of the function $G\left(\alpha_{0}(t), A_{0}, t+t_{0}\right)$. By construction $M\left(t_{0}\right)$ is periodic in $t_{0}$. With the terminology introduced above, $\varepsilon$ is the perturbation parameter and $t_{0}$ is the initial phase. 
The following scenario arises.

- If $M\left(t_{0}\right)$ has no zero, then there is no subharmonic solution, that is no periodic solution which continues the unperturbed one at $\varepsilon \neq 0$.

- Otherwise, if $M\left(t_{0}\right)$ has zeroes, the following two cases are possible: either $M\left(t_{0}\right)$ has a zero of finite order $\mathfrak{n}$ or $M\left(t_{0}\right)$ vanishes with all its derivatives. In the second case, because of analyticity, the function $M\left(t_{0}\right)$ is identically zero.

- If $M\left(t_{0}\right)$ has a simple zero (i.e. $\mathfrak{n}=1$ ), then the usual Melnikov's theory applies. In particular there exists at least one subharmonic solution, and it is analytic in the perturbation parameter $\varepsilon$.

- If $M\left(t_{0}\right)$ has a zero of order $\mathfrak{n}$, then in general no result can be given about the existence of subharmonic solutions. However one can introduce an infinite sequence of polynomial equations, which are defined iteratively: if the first equation admits a real non-zero root and all the following equations admit a real root, then a subharmonic solution exists, and it is a function analytic in suitable fractional power of $\varepsilon$; more precisely it is analytic in $\eta=\varepsilon^{1 / p}$, for some $p \leq \mathfrak{n}$ !, and hence it is analytic in $\varepsilon^{1 / \mathfrak{n} !}$. If at some step the root is simple, an algorithm can be given in order to construct recursively all the coefficients of the series.

- If we further assume that the order $\mathfrak{n}$ of the zero is odd, then we have that all the equations of the sequence satisfy the request made above on the roots, so that we can conclude that in such a case at least one subharmonic solution exists. Again, in order to really construct the solution, by providing an explicit recursive algorithm, we need that at a certain level of the iteration scheme a simple root appears.

- Moreover we have at most $\mathfrak{n}$ periodic solutions bifurcating from the unperturbed one with initial phase $t_{0}$. Of course, to count all subharmonic solutions we have also to sum over all the zeroes of the subharmonic Melnikov function.

- Finally, if $M\left(t_{0}\right)$ vanishes identically as a function of $t_{0}$, then we have to extend the analysis up to second order, and all the cases discussed above for $M\left(t_{0}\right)$ have to repeated for a suitable function $M_{1}\left(t_{0}\right)$, which is obtained in the following way. If $M\left(t_{0}\right) \equiv 0$ then the solution $t \rightarrow$ $(\alpha(t), A(t))$ is defined up to first order - as it is easy to check -, so that one can expand the function $G\left(\alpha(t), A(t), t+t_{0}\right)$ up to first order: we call $M_{1}\left(t_{0}\right)$ its average over a period of the unperturbed solution. In particular if also $M_{1}\left(t_{0}\right)$ vanishes identically then one can push the perturbation theory up to second order, and, after expanding the function $G\left(\alpha(t), A(t), t+t_{0}\right)$ up to second order, one defines $M_{2}\left(t_{0}\right)$ as its average over a period, and so on.

The first conclusion we can draw is that in general we cannot say that for any vector field $(F, G)$ there is at least one subharmonic solution of given period. We need some condition on $G$. We can require for $G$ to be a zero-mean function, so that it has at least one zero of odd order. For instance, this holds true if the vector field is Hamiltonian, since in such a case $G$ is the $\alpha$-derivative of a suitable function. The same result follows if the equations describe a Hamiltonian system in the presence of small friction - how small depends on the particular resonance one is looking at 6 . But of course, all these conditions are stronger than what is really needed.

A second conclusion is that, even when a subharmonic solution turns out to exist (and to be analytic in a suitable fractionary power of the perturbation parameter), a constructive algortithm to compute it within any given accuracy cannot be provided in general. This becomes possible only if some further assumption is made. So there are situations where one can obtain an existence result of the solution, but the solutin itself cannot be constructed. Note that such situations are highly non-generic, because they 
arise if one finds at each iterative step a polynomial with multiple roots - which is a non-generic case; cf. Appendix A.

The methods we shall use to prove the results above will be of two different types. We shall rely on standard general techniques, based on the Weierstrass preparation theorem, in order to show that under suitable assumptions the solutions exist and to prove in this case the convergence of the series. Moreover, we shall use a combination of the Newton-Puiseux process and the diagrammatic techniques based on the tree formalism 4, 5, 6 in order to provide a recursive algorithm, when possible. Note that in such a case the convergence of the Puiseux series follows by explicit construction of the coefficients, and an explicit bound of the radius of convergence is obtained through the estimates of the coefficients - on the contrary there is no way to provide quantitative bounds with the aforementioned abstract arguments. These results extend those in [6], where a special case was considered.

The paper is organised as follows. In Section 2 we formulate rigorously the problem of subharmonic bifurcations for analytic ordinary differential equations in the plane, and show that, if the subharmonic Melnikov function admits a finite order zero, the problem can be reduced to an analytic implicit equation problem - analyticity will be proved in Section 4 by using the tree formalism. In Section 3 we discuss the Newton-Puiseux process, which will be used to iteratively attack the problem. At each iteration step one has to solve a polynomial equation. Thus, in the complex setting [2] the process can be pushed forward indefinitely, whereas in the real setting one has to impose at each step that a real root exists. If the order of zero of the subharmonic Melnikov function is odd, the latter condition is automatically satisfied, and hence the existence of at least one subharmonic solution is obtained (Theorem (1). If at some step of the iteration a simple root appears, then we can give a fully constructive algorithm which allows us to estimate the radius of analyticity and to approximate the solution within any fixed accuracy (Theorem 21). This second result will be proved in Sections 5 and 6, again by relying on the tree formalism; some more technical aspects of the proof will be dealt with in Appendix B Finally in Section 7 we consider the case in which the subharmonic Melnikov function vanishes identically, so that one has to repeat the analysis for suitable higher order generalisations of that function. This will lead to Theorems 3 and 4 , which generalise Theorems 2 and 1, respectively.

\section{Set-up}

Let us consider the ordinary differential equation

$$
\left\{\begin{array}{l}
\dot{\alpha}=\omega(A)+\varepsilon F(\alpha, A, t), \\
\dot{A}=\varepsilon G(\alpha, A, t),
\end{array}\right.
$$

where $(\alpha, A) \in \mathcal{M}:=\mathbb{T} \times W$, with $W \subset \mathbb{R}$ an open set, the map $A \mapsto \omega(A)$ is real analytic in $A$, and the functions $F, G$ depend analytically on their arguments and are $2 \pi$-periodic in $\alpha$ and $t$. Finally $\varepsilon$ is a real parameter.

Set $\alpha_{0}(t)=\omega\left(A_{0}\right) t$ and $A_{0}(t)=A_{0}$. In the extended phase-space $\mathcal{M} \times \mathbb{R}$, for $\varepsilon=0$, the solution $\left(\alpha_{0}(t), A_{0}(t), t+t_{0}\right)$ describes an invariant torus, which is uniquely determined by the "energy" $A_{0}$. Hence the motion of the variables $(\alpha, A, t)$ is quasi-periodic, and reduces to a periodic motion whenever $\omega\left(A_{0}\right)$ becomes commensurate with 1 . If $\omega\left(A_{0}\right)$ is rational we say that the torus is resonant. The parameter $t_{0}$ will be called the initial phase: it fixes the initial datum on the torus. Only for some values of the parameter $t_{0}$ periodic solutions lying on the torus are expected to persist under perturbation: such solutions are called subharmonic solutions.

Denote by $T_{0}\left(A_{0}\right)=2 \pi / \omega\left(A_{0}\right)$ the period of the trajectories on the unperturbed torus, and define $\omega^{\prime}(A):=\mathrm{d} \omega(A) / \mathrm{d} A$. If $\omega\left(A_{0}\right)=p / q \in \mathbb{Q}$, call $T=T\left(A_{0}\right)=2 \pi q$ the period of the trajectories in the extended phase space. We shall call $p / q$ the order of the corresponding subharmonic solutions. 
Hypothesis 1. One has $\omega^{\prime}\left(A_{0}\right) \neq 0$.

Define

$$
M\left(t_{0}\right):=\frac{1}{T} \int_{0}^{T} \mathrm{~d} t G\left(\alpha_{0}(t), A_{0}, t+t_{0}\right),
$$

which is called the subharmonic Melnikov function of order $q / p$. Note that $M\left(t_{0}\right)$ is $2 \pi$-periodic in $t_{0}$.

Hypothesis 2. There exist $t_{0} \in[0,2 \pi)$ and $\mathfrak{n} \in \mathbb{N}$ such that

$$
\frac{\mathrm{d}^{k}}{\mathrm{~d} t_{0}^{k}} M\left(t_{0}\right)=0 \quad \forall 0 \leq k \leq \mathfrak{n}-1, \quad D\left(t_{0}\right):=\frac{\mathrm{d}^{\mathfrak{n}}}{\mathrm{d} t_{0}^{\mathfrak{n}}} M\left(t_{0}\right) \neq 0,
$$

that is $t_{0}$ is a zero of order $\mathfrak{n}$ for the subharmonic Melnikov function.

For notational semplicity, we shall not make explicit the dependence on $t_{0}$ most of times; for instance we shall write $D\left(t_{0}\right)=D$. For any $T$-periodic function $F$ we shall denote by $\langle F\rangle$ its average over the period $T$.

The solution of (2.1) with initial conditions $(\alpha(0), A(0))$ can be written as

$$
\left(\begin{array}{c}
\alpha(t) \\
A(t)
\end{array}\right)=W(t)\left(\begin{array}{c}
\alpha(0) \\
A(0)
\end{array}\right)+W(t) \int_{0}^{t} \mathrm{~d} \tau W^{-1}(\tau)\left(\begin{array}{c}
\Phi(\tau) \\
\Gamma(\tau)
\end{array}\right),
$$

where we have denoted by

$$
W(t)=\left(\begin{array}{cc}
1 & \omega^{\prime}\left(A_{0}\right) t \\
0 & 1
\end{array}\right)
$$

the Wronskian matrix solving the linearised system, and set

$$
\Phi(t)=\varepsilon F(t)+\omega(A(t))-\omega\left(A_{0}\right)-\omega^{\prime}\left(A_{0}\right)\left(A(t)-A_{0}\right), \quad \Gamma(t)=\varepsilon G(t) .
$$

shortening $F(t)=F\left(\alpha(t), A(t), t+t_{0}\right)$ and $G(t)=G\left(\alpha(t), A(t), t+t_{0}\right)$.

By using explicitly (2.5) in (2.4) we obtain

$$
\left\{\begin{array}{l}
\alpha(t)=\alpha(0)+t \omega^{\prime}\left(A_{0}\right) A(0)+\int_{0}^{t} \mathrm{~d} \tau \Phi(\tau)+\omega^{\prime}\left(A_{0}\right) \int_{0}^{t} \mathrm{~d} \tau \int_{0}^{\tau} \mathrm{d} \tau^{\prime} \Gamma\left(\tau^{\prime}\right), \\
A(t)=A(0)+\int_{0}^{t} \mathrm{~d} \tau \Gamma(\tau),
\end{array}\right.
$$

with the notations (2.6).

In order to obtain a periodic solution we need for the mean $\langle\Gamma\rangle$ of the function $\Gamma$ to be zero. In this case, if we fix also

$$
A(0)=-\frac{1}{\omega^{\prime}\left(A_{0}\right)}\langle\Phi\rangle-\langle\mathcal{G}\rangle, \quad \mathcal{G}(\tau)=\int_{0}^{\tau} \mathrm{d} \tau^{\prime}\left(\Gamma\left(\tau^{\prime}\right)-\langle\Gamma\rangle\right),
$$

then the corresponding solution turns out to be periodic. So, instead of (2.7), we consider the system

$$
\left\{\begin{array}{l}
\alpha(t)=\alpha(0)+\int_{0}^{t} \mathrm{~d} \tau(\Phi(\tau)-\langle\Phi\rangle)+\omega^{\prime}\left(A_{0}\right) \int_{0}^{t} \mathrm{~d} \tau(\mathcal{G}(\tau)-\langle\mathcal{G}\rangle) \\
A(t)=A(0)+\mathcal{G}(t) \\
\langle\Gamma\rangle=0
\end{array}\right.
$$

where $A(0)$ is determined according to (2.8) and $\alpha(0)$ is considered as a free parameter. 
We start by considering the auxiliary system

$$
\left\{\begin{array}{l}
\alpha(t)=\alpha(0)+\int_{0}^{t} \mathrm{~d} \tau(\Phi(\tau)-\langle\Phi\rangle)+\omega^{\prime}\left(A_{0}\right) \int_{0}^{t} \mathrm{~d} \tau(\mathcal{G}(\tau)-\langle\mathcal{G}\rangle), \\
A(t)=A(0)+\mathcal{G}(t),
\end{array}\right.
$$

that is we neglect for the moment the condition that the mean of $\Gamma$ has to be zero. Of course, only in that case the solution of (2.10) is solution also of (2.9), hence of (2.7).

It can be more convenient to work in Fourier space. As we are looking for periodic solutions of period $T=2 \pi q$, i.e. of frequency $\omega=1 / q$, we can write

$$
\alpha(t)=\alpha_{0}(t)+\beta(t), \quad \beta(t)=\sum_{\nu \in \mathbb{Z}} \mathrm{e}^{i \omega \nu t} \beta_{\nu}, \quad A(t)=A_{0}+B(t), \quad B(t)=\sum_{\nu \in \mathbb{Z}} \mathrm{e}^{i \omega \nu t} B_{\nu} .
$$

If we expand

$$
G\left(\alpha, A, t+t_{0}\right)=\sum_{\nu \in \mathbb{Z}} \sum_{\nu^{\prime} \in \mathbb{Z}} \mathrm{e}^{i \nu \alpha+i \nu^{\prime}\left(t+t_{0}\right)} G_{\nu, \nu^{\prime}}(A), \quad G_{\nu, \nu^{\prime}}\left(A, t_{0}\right):=\mathrm{e}^{i \nu^{\prime} t_{0}} G_{\nu, \nu^{\prime}}(A),
$$

with an analogous expressions for the function $\Phi(t)$, then we can write

$$
\Gamma(t)=\sum_{\nu \in \mathbb{Z}} \mathrm{e}^{i \omega \nu t} \Gamma_{\nu}, \quad \Phi(t)=\sum_{\nu \in \mathbb{Z}} \mathrm{e}^{i \omega \nu t} \Phi_{\nu}
$$

with

$$
\begin{aligned}
\Gamma_{\nu} & =\varepsilon \sum_{r=0}^{\infty} \sum_{s=0}^{\infty} \sum_{p \nu_{0}+q \nu_{0}^{\prime}+\nu_{1}+\ldots+\nu_{r+s}=\nu} \frac{1}{r ! s !}\left(i \nu_{0}\right)^{r} \partial_{A}^{s} G_{\nu_{0}, \nu_{0}^{\prime}}\left(A_{0}, t_{0}\right) \beta_{\nu_{1}} \ldots \beta_{\nu_{r}} B_{\nu_{r+1}} \ldots B_{\nu_{r+s}}, \\
\Phi_{\nu} & =\varepsilon \sum_{r=0}^{\infty} \sum_{s=0}^{\infty} \sum_{p \nu_{0}+q \nu_{0}^{\prime}+\nu_{1}+\ldots+\nu_{r+s}=\nu} \frac{1}{r ! s !}\left(i \nu_{0}\right)^{r} \partial_{A}^{s} F_{\nu_{0}, \nu_{0}^{\prime}}\left(A_{0}, t_{0}\right) \beta_{\nu_{1}} \ldots \beta_{\nu_{r}} B_{\nu_{r+1}} \ldots B_{\nu_{r+s}} \\
& +\sum_{s=2}^{\infty} \sum_{\nu_{1}+\ldots+\nu_{s}=\nu} \frac{1}{s !} \partial_{A}^{s} \omega\left(A_{0}\right) B_{\nu_{1}} \ldots B_{\nu_{s}} .
\end{aligned}
$$

Then (2.10) becomes

$$
\left\{\begin{array}{l}
\beta_{\nu}=\frac{\Phi_{\nu}}{i \omega \nu}+\omega^{\prime}\left(A_{0}\right) \frac{\Gamma_{\nu}}{(i \omega \nu)^{2}} \\
B_{\nu}=\frac{\Gamma_{\nu}}{i \omega \nu}
\end{array}\right.
$$

for $\nu \neq 0$, provided

$$
\left\{\begin{array}{l}
\beta_{0}=\alpha(0)-\sum_{\substack{\nu \in \mathbb{Z} \\
\nu \neq 0}} \frac{\Phi_{\nu}}{i \omega \nu}-\omega^{\prime}\left(A_{0}\right) \sum_{\substack{\nu \in \mathbb{Z} \\
\nu \neq 0}} \frac{\Gamma_{\nu}}{(i \omega \nu)^{2}} \\
B_{0}=A(0)-\sum_{\substack{\nu \in \mathbb{Z} \\
\nu \neq 0}} \frac{\Gamma_{\nu}}{i \omega \nu}=-\frac{\Phi_{0}}{\omega^{\prime}\left(A_{0}\right)}
\end{array}\right.
$$

for $\nu=0$. Also (2.9) can be written in the same form, with the further constraint $\Gamma_{0}=0$.

Then we can use $\beta_{0}$ as a free parameter, instead of $\alpha(0)$. This means that we look for a value of $\beta_{0}$ (depending on $\varepsilon$ ) such that, by defining $B_{0}$ according to the second equation in (2.16), the coefficients 
$\beta_{\nu}, B_{\nu}$ are given by (2.15) for $\nu \neq 0$. In other words, in Fourier space (2.10) becomes

$$
\left\{\begin{array}{l}
\beta_{\nu}=\frac{\Phi_{\nu}}{i \omega \nu}+\omega^{\prime}\left(A_{0}\right) \frac{\Gamma_{\nu}}{(i \omega \nu)^{2}}, \quad B_{\nu}=\frac{\Gamma_{\nu}}{i \omega \nu}, \quad \nu \neq 0, \\
B_{0}=-\frac{\Phi_{0}}{\omega^{\prime}\left(A_{0}\right)},
\end{array}\right.
$$

whereas $\beta_{0}$ is left as a free parameter.

We look for a solution $(\bar{\alpha}(t), \bar{A}(t))$ of (2.10) which can be written as a formal Taylor series in $\varepsilon$ and $\beta_{0}$, so that

$$
\begin{aligned}
& \bar{\alpha}(t)=\bar{\alpha}\left(t ; \varepsilon, \beta_{0}\right)=\alpha_{0}(t)+\sum_{k=1}^{\infty} \sum_{j=0}^{\infty} \varepsilon^{k} \beta_{0}^{j} \bar{\beta}^{(k, j)}(t), \\
& \bar{A}(t)=\bar{A}\left(t ; \varepsilon, \beta_{0}\right)=A_{0}+\sum_{k=1}^{\infty} \sum_{j=0}^{\infty} \varepsilon^{k} \beta_{0}^{j} \bar{B}^{(k, j)}(t),
\end{aligned}
$$

which reduces to $\left(\alpha_{0}(t), A_{0}\right)$ as $\varepsilon \rightarrow 0$. By comparing (2.18) with (2.11) we can write the Fourier coefficients of the solution $(\bar{\alpha}(t), \bar{A}(t))$, for $\nu \neq 0$, as

$$
\bar{\beta}_{\nu}=\bar{\beta}_{\nu}\left(\varepsilon, \beta_{0}\right)=\sum_{k=1}^{\infty} \sum_{j=0}^{\infty} \varepsilon^{k} \beta_{0}^{j} \bar{\beta}_{\nu}^{(k, j)}, \quad \bar{B}_{\nu}=\bar{B}_{\nu}\left(\varepsilon, \beta_{0}\right)=\sum_{k=1}^{\infty} \sum_{j=0}^{\infty} \varepsilon^{k} \beta_{0}^{j} \bar{B}_{\nu}^{(k, j)},
$$

and, analogously, $\bar{B}_{0}^{(k, j)}$ is the contribution to order $k$ in $\varepsilon$ and $j$ in $\beta_{0}$ to $\bar{B}_{0}$.

By analyticity also the function $\bar{\Gamma}(t)=\varepsilon G\left(\bar{\alpha}(t), \bar{A}(t), t+t_{0}\right)$ can be formally expanded in powers of $\varepsilon$ and $\beta_{0}$, and one has

$$
\bar{\Gamma}(t)=\bar{\Gamma}\left(t ; \varepsilon, \beta_{0}\right)=\sum_{k=1}^{\infty} \sum_{j=0}^{\infty} \varepsilon^{k} \beta_{0}^{j} \bar{\Gamma}^{(k, j)}(t)=\sum_{k=1}^{\infty} \sum_{j=0}^{\infty} \varepsilon^{k} \beta_{0}^{j} \sum_{\nu \in \mathbb{Z}} \mathrm{e}^{i \omega \nu t} \bar{\Gamma}_{\nu}^{(k, j)},
$$

where each $\bar{\Gamma}_{\nu}^{(k, j)}$ is expressed in terms of the Taylor coefficients of (2.19) of order strictly less than $k, j$. By definition one has $\left\langle\bar{\Gamma}^{(k, j)}\right\rangle=\bar{\Gamma}_{0}^{(k, j)}$. The same considerations hold for $\bar{\Phi}(t)=\Phi\left(\bar{\alpha}(t), \bar{A}(t), t+t_{0}\right)$.

Hence one can formally write, for all $k \geq 1$ and $j \geq 0$

$$
\left\{\begin{array}{l}
\bar{\beta}_{\nu}^{(k, j)}=\frac{\bar{\Phi}_{\nu}^{(k, j)}}{i \omega \nu}+\omega^{\prime}\left(A_{0}\right) \frac{\bar{\Gamma}_{\nu}^{(k, j)}}{(i \omega \nu)^{2}}, \quad \bar{B}_{\nu}^{(k, j)}=\frac{\bar{\Gamma}_{\nu}^{(k, j)}}{i \omega \nu}, \quad \nu \neq 0 \\
\bar{B}_{0}^{(k, j)}=-\frac{\bar{\Phi}_{0}^{(k, j)}}{\omega^{\prime}\left(A_{0}\right)} .
\end{array}\right.
$$

Lemma 1. For any $\beta_{0} \in \mathbb{R}$ the system (2.10) admits a solution $(\bar{\alpha}(t), \bar{A}(t))$ which is T-periodic in time and analytic in $\varepsilon$, depending analytically on the parameter $\beta_{0}$.

Proof. One can use the tree formalism introduced in Section 4 see in particular Proposition 1 .

It can be convenient to introduce also the Taylor coefficients

$$
\begin{array}{llrl}
\bar{\beta}_{\nu}^{(k)}\left(\beta_{0}\right) & =\sum_{j \geq 0} \beta_{0}^{j} \bar{\beta}_{\nu}^{(k, j)}, & \bar{B}_{\nu}^{(k)}\left(\beta_{0}\right) & =\sum_{j \geq 0} \beta_{0}^{j} \bar{B}_{\nu}^{(k, j)}, \\
\bar{\Gamma}_{\nu}^{(k)}\left(\beta_{0}\right) & =\sum_{j \geq 0} \beta_{0}^{j} \bar{\Gamma}_{\nu}^{(k, j)}, & \bar{\Phi}_{\nu}^{(k)}\left(\beta_{0}\right) & =\sum_{j \geq 0} \beta_{0}^{j} \bar{\Phi}_{\nu}^{(k, j)},
\end{array}
$$

Note that $\bar{\Gamma}_{\nu}^{(k)}(0)=\bar{\Gamma}_{\nu}^{(k, 0)}$, and so on. 
Lemma 2. Consider the system (2.10). Assume that $\bar{\Gamma}_{0}^{(k)}(0)=0$ for all $k \in \mathbb{N}$. Then for $\beta_{0}=0$ the solution $(\bar{\alpha}(t), \bar{A}(t))$ of (2.10) is also a solution of (2.9)).

Proof. Simply note that (2.10) reduces to (2.9) if $\bar{\Gamma}_{0}^{(k)}(0)=0$ for all $k \in \mathbb{N}$.

Of course we expect in general that $\bar{\Gamma}_{0}^{(k)}(0)$ do not vanish for all $k \in \mathbb{N}$. In that case, let $k_{0} \in \mathbb{N}$ be such that $\bar{\Gamma}_{0}^{(k)}(0)=0$ for $k=1, \ldots, k_{0}$ and $\bar{\Gamma}_{0}^{\left(k_{0}+1\right)}(0) \neq 0$.

Let us define

$$
\mathcal{F}^{(0)}\left(\varepsilon, \beta_{0}\right):=\sum_{k, j \geq 0} \varepsilon^{k} \beta_{0}^{j} \mathcal{F}_{k, j}^{(0)}, \quad \mathcal{F}_{k, j}^{(0)}=\bar{\Gamma}_{0}^{(k+1, j)},
$$

so that $\varepsilon \mathcal{F}^{(0)}\left(\varepsilon, \beta_{0}\right)=\left\langle\bar{\Gamma}\left(\cdot ; \varepsilon, \beta_{0}\right)\right\rangle$.

Lemma 3. $\mathcal{F}^{(0)}\left(\varepsilon, \beta_{0}\right)$ is $\beta_{0}$-general of order $\mathfrak{n}$, i.e. $\mathcal{F}_{0, j}^{(0)}=0$ for $j=0, \ldots, \mathfrak{n}-1$, while $\mathcal{F}_{0, \mathfrak{n}}^{(0)} \neq 0$.

Proof. This can be easily shown using the tree formalism introduced in Section 5 . In fact for all $j$, $\mathcal{F}_{0, j}^{(0)}=\bar{\Gamma}_{0}^{(1, j)}$ is associated with a tree with 1 node and $j$ leaves. Hence one has

$$
j ! \bar{\Gamma}_{0}^{(1, j)}=\left\langle\partial_{\alpha}^{j} G\left(\alpha_{0}(\cdot), A_{0}, \cdot+t_{0}\right)\right\rangle=\left(-\omega\left(A_{0}\right)\right)^{-j} \frac{\mathrm{d}^{j} M}{\mathrm{~d} t_{0}^{j}}\left(t_{0}\right),
$$

where the second equality is provided by Lemma 3.9 on [6]. Then $\mathcal{F}^{(0)}\left(\varepsilon, \beta_{0}\right)$ is $\beta_{0}$-general of order $\mathfrak{n}$ by Hypothesis 2

Our aim is to find $\beta_{0}=\beta_{0}(\varepsilon)$ such that $\mathcal{F}^{(0)}\left(\varepsilon, \beta_{0}(\varepsilon)\right) \equiv 0$. For such $\beta_{0}$ a solution of (2.10) is also solution of (2.9). If we are successful in doing so, then we have proved the existence of subharmonic solutions.

\section{The Newton-Puiseux process and main results}

Given a convergent power series $\mathcal{F}^{(0)}\left(\varepsilon, \beta_{0}\right) \in \mathbb{R}\left\{\varepsilon, \beta_{0}\right\}$ as in (2.23), we call carrier of $\mathcal{F}^{(0)}$ the set

$$
\Delta\left(\mathcal{F}^{(0)}\right):=\left\{(k, j) \in \mathbb{N} \times \mathbb{N}: \mathcal{F}_{k, j}^{(0)} \neq 0\right\} .
$$

For all $v \in \Delta\left(\mathcal{F}^{(0)}\right)$ let us consider the positive quadrant $\mathfrak{A}_{v}:=\{v\}+\left(\mathbb{R}_{+}\right)^{2}$ moved up to $v$, and define

$$
\mathfrak{A}:=\bigcup_{v \in \Delta\left(\mathcal{F}^{(0)}\right)} \mathfrak{A}_{v}
$$

Let $\mathcal{C}$ be the convex hull of $\mathfrak{A}$. The boundary $\partial \mathcal{C}$ consists of a compact polygonal path $\mathcal{P}^{(0)}$ and two half lines $\mathcal{R}_{1}^{(0)}$ and $\mathcal{R}_{2}^{(0)}$. The polygonal path $\mathcal{P}^{(0)}$ is called the Newton polygon of $\mathcal{F}^{(0)}$.

Notice that if the Newton polygon is a single point or, more generally, if $\mathcal{F}_{k, 0}^{(0)}=0$ for all $k \geq 0$ then there exists $\overline{\mathrm{J}} \geq 1$ such that $\mathcal{F}^{(0)}\left(\varepsilon, \beta_{0}\right)=\beta_{0}^{\bar{\jmath}} \cdot \overline{\mathcal{G}}\left(\varepsilon, \beta_{0}\right)$ with $\overline{\mathcal{G}}(\varepsilon, 0) \neq 0$, hence $\beta_{0} \equiv 0$ is a solution of equation $\mathcal{F}^{(0)}(\varepsilon, 0)=0$, that is the conclusion of Lemma 2. Otherwise, if we further assume that $\mathcal{F}^{(0)}$ is $\beta_{0}$-general of some finite order $\mathfrak{n}$, there is at least a point of $\Delta\left(\mathcal{F}^{(0)}\right)$ on each axis, then the Newton polygon $\mathcal{P}^{(0)}$ is formed by $N_{0} \geq 1$ segments $\mathcal{P}_{1}^{(0)}, \ldots, \mathcal{P}_{N_{0}}^{(0)}$ and we write $\mathcal{P}^{(0)}=\mathcal{P}_{1}^{(0)} \cup \ldots \cup \mathcal{P}_{N_{0}}^{(0)}$; cf. Figure 1 


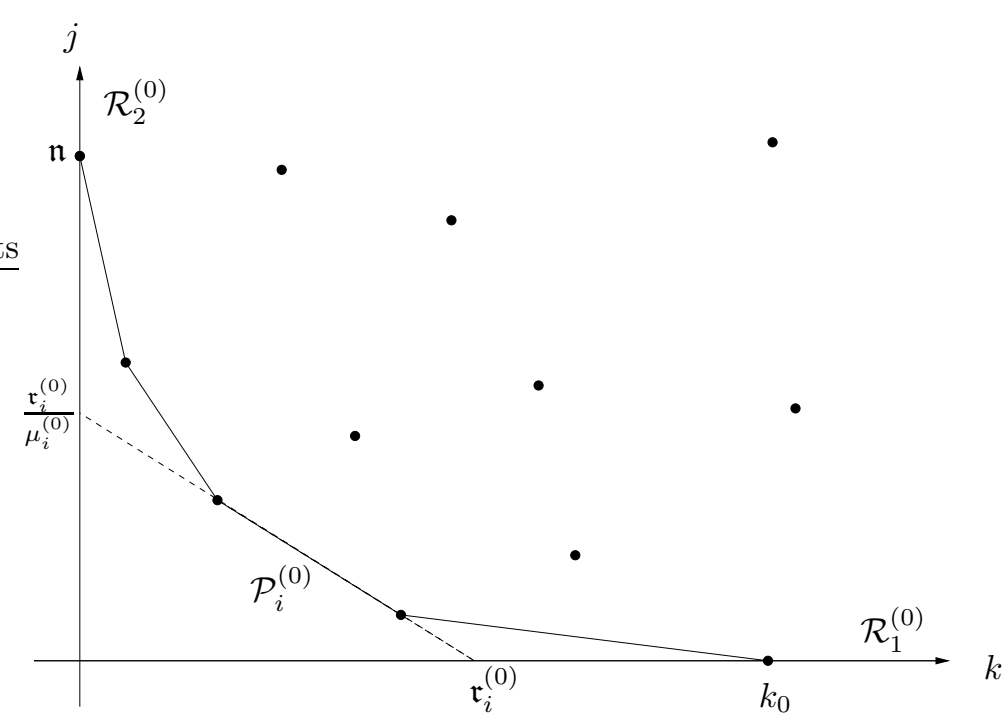

Figure 1: Newton polygon.

For all $i=1, \ldots, N_{0}$ let $-1 / \mu_{i}^{(0)} \in \mathbb{Q}$ be the slope of the segment $\mathcal{P}_{i}^{(0)}$, so that one can partition $\mathcal{F}^{(0)}$ according to the weights given by $\mu_{i}^{(0)}$ :

$$
\mathcal{F}^{(0)}\left(\varepsilon, \beta_{0}\right)=\widetilde{\mathcal{F}}_{i}^{(0)}\left(\varepsilon, \beta_{0}\right)+\mathcal{G}_{i}^{(0)}\left(\varepsilon, \beta_{0}\right)=\sum_{k+j \mu_{i}^{(0)}=\mathbf{r}_{i}^{(0)}} \mathcal{F}_{k, j}^{(0)} \varepsilon^{k} \beta_{0}^{j}+\sum_{k+j \mu_{i}^{(0)}>\mathfrak{r}_{i}^{(0)}} \mathcal{F}_{k, j}^{(0)} \varepsilon^{k} \beta_{0}^{j},
$$

where $\mathfrak{r}_{i}^{(0)}$ is the intercept on the $k$-axis of the continuation of $\mathcal{P}_{i}^{(0)}$.

Hence the first approximate solutions of $\mathcal{F}\left(\varepsilon, \beta_{0}\right)=0$ are the solutions of the quasi-homogeneous equations

$$
\widetilde{\mathcal{F}}_{i}^{(0)}\left(\varepsilon, \beta_{0}\right)=\sum_{k \mathfrak{p}_{i}^{(0)}+j \mathfrak{h}_{i}^{(0)}=\mathfrak{s}_{i}^{(0)}} \mathcal{F}_{k, j}^{(0)} \varepsilon^{k} \beta_{0}^{j}=0, \quad i=1, \ldots, N_{0},
$$

where $\mathfrak{h}_{i}^{(0)} / \mathfrak{p}_{i}^{(0)}=\mu_{i}^{(0)}$, with $\mathfrak{h}_{i}^{(0)}, \mathfrak{p}_{i}^{(0)}$ relatively prime integers, and $\mathfrak{s}_{i}^{(0)}=\mathfrak{p}_{i}^{(0)} \mathfrak{r}_{i}^{(0)}$.

We introduce the polynomials $P_{i}^{(0)}=P_{i}^{(0)}(c)$ in such a way that

$$
\widetilde{\mathcal{F}}_{i}^{(0)}\left(\varepsilon, c\left(\sigma_{0} \varepsilon\right)^{\mu_{i}^{(0)}}\right)=\left(\sigma_{0} \varepsilon\right)^{\mathfrak{r}_{i}^{(0)}} \sum_{k \mathfrak{p}_{i}^{(0)}+j \mathfrak{h}_{i}^{(0)}=\mathfrak{s}_{i}^{(0)}} Q_{k, j} c^{j}=\left(\sigma_{0} \varepsilon\right)^{\mathfrak{r}_{i}^{(0)}} P_{i}^{(0)}(c), \quad \sigma_{0}:=\operatorname{sign}(\varepsilon),
$$

where $Q_{k, j}=\mathcal{F}_{k, j}^{(0)} \sigma_{0}^{k}$.

Lemma 4. With the notation introduced before, let $\Pi_{i}^{(0)}$ be the projection of the segment $\mathcal{P}_{i}^{(0)}$ on the $j$-axis and let $\ell_{i}=\ell\left(\Pi_{i}^{(0)}\right)$ be the length of $\Pi_{i}^{(0)}$. Then $P_{i}^{(0)}(c)$ has $\ell_{i}$ complex non-zero roots counting multiplicity.

Proof. Let $m, n$ be respectively the maximum and the minimum among the exponents of the variable $\beta_{0}$ in $\widetilde{\mathcal{F}}_{i}^{(0)}$. Then $\ell_{i}=m-n$. Hence $P_{i}^{(0)}$ is a polynomial of degree $m$ and minimum power $n$ : we can write $P_{i}^{(0)}(c)=c^{n} \widetilde{P}(c)$, where $\widetilde{P}$ has degree $\ell_{i}$ and $\widetilde{P}(0) \neq 0$. Fundamental theorem of algebra guarantees that $\widetilde{P}(c)=0$ has $\ell_{i}$ complex solutions counting multiplicity, which are all the non-zero roots of $P_{i}^{(0)}$. 
Let $\Re_{0}$ be the set of all the non-zero real solutions of the polynomial equations $P_{i}^{(0)}(c)=0$. If $\Re_{0}=\emptyset$ the system (2.1) has no subharmonic solution, as one can easily verify.

Let us suppose then that there exists $c_{0} \in \Re_{0}$, such that $c_{0}\left(\sigma_{0} \varepsilon\right)^{\mu_{i}^{(0)}}$ is a first approximate solution of the implicit equation $\mathcal{F}^{(0)}\left(\varepsilon, \beta_{0}\right)=0$ for a suitable $i=1, \ldots, N_{0}$. From now on we shall drop the label $i$ to lighten the notation. We now set $\varepsilon_{1}=\left(\sigma_{0} \varepsilon\right)^{1 / \mathfrak{p}^{(0)}}$, and, as $\varepsilon_{1}^{\mathfrak{s}^{(0)}} \operatorname{divides} \mathcal{F}^{(0)}\left(\sigma_{0} \varepsilon_{1}^{\mathfrak{p}^{(0)}}, c_{0} \varepsilon_{1}^{\mathfrak{h}^{(0)}}+y_{1} \varepsilon_{1}^{\mathfrak{h}^{(0)}}\right)$, we obtain a new power series $\mathcal{F}^{(1)}\left(\varepsilon_{1}, y_{1}\right)$ given by

$$
\mathcal{F}^{(0)}\left(\sigma_{0} \varepsilon_{1}^{\mathfrak{p}^{(0)}}, c_{0} \varepsilon_{1}^{\mathfrak{h}^{(0)}}+y_{1} \varepsilon_{1}^{\mathfrak{h}^{(0)}}\right)=\varepsilon_{1}^{\mathfrak{s}^{(0)}} \mathcal{F}^{(1)}\left(\varepsilon_{1}, y_{1}\right)
$$

which is $y_{1}$-general of order $\mathfrak{n}_{1}$ for some $\mathfrak{n}_{1} \geq 1$.

Lemma 5. With the notations introduced before, let us write $P^{(0)}(c)=g_{0}(c)\left(c-c_{0}\right)^{m_{0}}$ with $g_{0}\left(c_{0}\right) \neq 0$ and $m_{0} \leq \mathfrak{n}$. Then $\mathfrak{n}_{1}=m_{0}$.

Proof. This simply follows by the definitions of $\mathcal{F}^{(1)}$ and $P^{(0)}$. In fact we have

$$
\varepsilon_{1}^{\mathfrak{s}^{(0)}} \mathcal{F}^{(1)}\left(\varepsilon_{1}, y_{1}\right)=\varepsilon_{1}^{\mathfrak{s}^{(0)}}\left(\sum_{k+\mu^{(0)}} Q_{k=\mathfrak{r}^{(0)}} Q_{k, j}\left(c_{0}+y_{1}\right)^{j}+\varepsilon_{1}(\ldots)\right)
$$

so that $\mathcal{F}^{(1)}\left(0, y_{1}\right)=P^{(0)}\left(c_{0}+y_{1}\right)=g_{0}\left(c_{0}+y_{1}\right) y_{1}^{m_{0}}$, and $g_{0}\left(c_{0}+y_{1}\right) \neq 0$ for $y_{1}=0$. Hence $\mathcal{F}^{(1)}$ is $y_{1}$-general of order $\mathfrak{n}_{1}=m_{0}$.

Now we restart the process just described: we construct the Newton polygon $\mathcal{P}^{(1)}$ of $\mathcal{F}^{(1)}$. If $\mathcal{F}_{k, 0}^{(1)}=0$ for all $k \geq 0$, then $\mathcal{F}^{(1)}\left(\varepsilon_{1}, 0\right) \equiv 0$, so that we have $\mathcal{F}^{(0)}\left(\varepsilon, c_{0}\left(\sigma_{0} \varepsilon\right)^{\mu^{(0)}}\right) \equiv 0$, i.e. $c_{0}\left(\sigma_{0} \varepsilon\right)^{\mu^{(0)}}$ is a solution of the implicit equation $\mathcal{F}^{(0)}\left(\varepsilon, \beta_{0}\right)=0$. Otherwise we consider the segments $\mathcal{P}_{1}^{(1)}, \ldots, \mathcal{P}_{N_{1}}^{(1)}$ with slopes $-1 / \mu_{i}^{(1)}$ for all $i=1, \ldots, N_{1}$, and we obtain

$$
\mathcal{F}^{(1)}\left(\varepsilon_{1}, y_{1}\right)=\widetilde{\mathcal{F}}_{i}^{(1)}\left(\varepsilon_{1}, y_{1}\right)+\mathcal{G}_{i}^{(1)}\left(\varepsilon_{1}, y_{1}\right)=\sum_{k+j \mu_{i}^{(1)}=\mathfrak{r}_{i}^{(1)}} \mathcal{F}_{k, j}^{(1)} \varepsilon_{1}^{k} y_{1}^{j}+\sum_{k+j \mu_{i}^{(1)}>\mathfrak{r}_{i}^{(1)}} \mathcal{F}_{k, j}^{(1)} \varepsilon_{1}^{k} y_{1}^{j},
$$

where $\mathfrak{r}_{i}^{(1)}$ is the intercept on the $k$-axis of the continuation of $\mathcal{P}_{i}^{(1)}$. Hence the first approximate solutions of $\mathcal{F}^{(1)}\left(\varepsilon_{1}, y_{1}\right)=0$ are the solutions of the quasi-homogeneous equations

$$
\widetilde{\mathcal{F}}_{i}^{(1)}\left(\varepsilon_{1}, y_{1}\right)=\sum_{k \mathfrak{p}_{i}^{(1)}+j \mathfrak{h}_{i}^{(1)}=\mathfrak{s}_{i}^{(1)}} \mathcal{F}_{k, j}^{(1)} \varepsilon_{1}^{k} y_{1}^{j}=0, \quad i=1, \ldots, N_{1}
$$

where $\mathfrak{h}_{i}^{(1)} / \mathfrak{p}_{i}^{(1)}=\mu_{i}^{(1)}$, with $\mathfrak{h}_{i}^{(1)}, \mathfrak{p}_{i}^{(1)}$ relatively prime integers, and $\mathfrak{s}_{i}^{(1)}=\mathfrak{p}_{i}^{(1)} \mathfrak{r}_{i}^{(1)}$

Thus we define the polynomials $P_{i}^{(1)}$ such that

$$
\widetilde{\mathcal{F}}_{i}^{(1)}\left(\varepsilon_{1}, c \varepsilon_{1}^{\mu_{i}^{(1)}}\right)=\varepsilon_{1}^{\mathfrak{r}_{i}^{(1)}} \sum_{k \mathfrak{p}_{i}^{(1)}+j \mathfrak{h}_{i}^{(1)}=\mathfrak{s}_{i}^{(1)}} \mathcal{F}_{k, j}^{(1)} c^{j}=\varepsilon_{1}^{\mathfrak{r}_{i}^{(1)}} P_{i}^{(1)}(c),
$$

and we call $\Re_{1}$ the set of the real roots of the polynomials $P_{i}^{(1)}$. If $\Re_{1}=\emptyset$, we stop the process as there is no subharmonic solution. Otherwise we call $P^{(1)}$ (i.e. again we omit the label $i$ ) the polynomial which has a real root $c_{1}$, so that $c_{1} \varepsilon_{1}^{\mu^{(1)}}$ is an approximate solution of the equation $\mathcal{F}^{(1)}\left(\varepsilon_{1}, y_{1}\right)=0$. Again we substitute $\varepsilon_{2}=\varepsilon_{1}^{1 / \mathfrak{p}^{(1)}}$, and we obtain

$$
\mathcal{F}^{(1)}\left(\varepsilon_{2}^{\mathfrak{p}^{(1)}}, c_{1} \varepsilon_{2}^{\mathfrak{h}^{(1)}}+y_{2} \varepsilon_{2}^{\mathfrak{h}^{(1)}}\right)=\varepsilon_{2}^{\mathfrak{s}^{(1)}} \mathcal{F}^{(2)}\left(\varepsilon_{2}, y_{2}\right),
$$


which is $y_{2}$-general of order $\mathfrak{n}_{2} \leq \mathfrak{n}_{1}$, and so on. Iterating the process we eventually obtain a sequence of approximate solutions

$$
\beta_{0}=\left(\sigma_{0} \varepsilon\right)^{\mu^{(0)}}\left(c_{0}+y_{1}\right), \quad y_{1}=\varepsilon_{1}^{\mu^{(1)}}\left(c_{1}+y_{2}\right), \quad y_{2}=\varepsilon_{2}^{\mu^{(2)}}\left(c_{2}+y_{3}\right), \quad \ldots
$$

where $c_{n}$ is a (real) root of the polynomial $P^{(n)}$ such that

$$
\mathcal{F}^{(n)}\left(\varepsilon_{n}, c \varepsilon_{n}^{\mu^{(n)}}\right)=\varepsilon_{n}^{\mathfrak{r}^{(n)}}\left(P^{(n)}(c)+\varepsilon_{n}(\ldots)\right),
$$

for all $n \geq 0$, where the functions $\mathcal{F}^{(n)}\left(\varepsilon_{n}, y_{n}\right)$ are defined recursively as $\mathcal{F}^{(n)}\left(\varepsilon_{n+1}^{\mathfrak{p}^{(n)}}, c_{n} \varepsilon_{n+1}^{\mathfrak{h}^{(n)}}+y_{n+1} \varepsilon_{n+1}^{\mathfrak{h}^{(n)}}\right)=$ $\varepsilon_{n+1}^{\mathfrak{s}^{(n)}} \mathcal{F}^{(n+1)}\left(\varepsilon_{n+1}, y_{n+1}\right)$ for $n \geq 1$, with $\varepsilon_{n+1}=\varepsilon_{n}^{1 / \mathfrak{p}^{(n)}}$ and the constants $\mu^{(n)}, \mathfrak{r}^{(n)}, \mathfrak{s}^{(n)}, \mathfrak{h}^{(n)}, \mathfrak{p}^{(n)}$ defined as in the case $n=0$ in terms of a segment $\mathcal{P}_{i}^{(n)}$ of the Newton polygon of $\mathcal{F}^{(n)}$. Therefore

$$
\beta_{0}=c_{0}\left(\sigma_{0} \varepsilon\right)^{\mu^{(0)}}+c_{1}\left(\sigma_{0} \varepsilon\right)^{\mu^{(0)}+\mu^{(1)} / \mathfrak{p}^{(0)}}+c_{2}\left(\sigma_{0} \varepsilon\right)^{\mu^{(0)}+\mu^{(1)} / \mathfrak{p}^{(0)}+\mu^{(2)} / \mathfrak{p}^{(0)} \mathfrak{p}^{(1)}}+\ldots
$$

is a formal expansion of $\beta_{0}$ as a series in ascending fractional powers of $\sigma_{0} \varepsilon$. This iterating method is called the Newton-Puiseux process. Of course this does not occur if we have $\Re_{n}=\emptyset$ at a certain step $n$-th, with $n \geq 0$.

From now on we shall suppose $\Re_{n} \neq \emptyset$ for all $n \geq 0$. Set also $\mathfrak{n}_{0}=\mathfrak{n}$.

Lemma 6. With the notation introduced before, if $\mathfrak{n}_{i+1}=d_{i}:=\operatorname{deg}\left(P^{(i)}\right)$ for some $i$, then $\mu^{(i)}$ is integer.

Proof. Without loss of generality we shall prove the result for the case $i=0$. Recall that

$$
\mathcal{F}^{(1)}\left(0, y_{1}\right)=\sum_{k+\mu^{(0)} j=\mathfrak{r}^{(0)}} Q_{k, j}\left(c_{0}+y_{1}\right)^{j}=P^{(0)}\left(c_{0}+y_{1}\right),
$$

with $\mathfrak{r}^{(0)}=\mu^{(0)} d_{0}$. If $d_{0}=\mathfrak{n}_{1}$, then $P^{(0)}$ is of the form $P^{(0)}(c)=R_{0}\left(c-c_{0}\right)^{\mathfrak{n}_{1}}$, with $R_{0} \neq 0$. In particular this means that $Q_{k, \mathfrak{n}_{1}-1} \neq 0$ for some integer $k \geq 0$ with the constraint $k+\mu^{(0)}\left(\mathfrak{n}_{1}-1\right)=\mu^{(0)} \mathfrak{n}_{1}$. Hence $\mu^{(0)}=k$ is integer.

Lemma 7. With the notations introduced before, there exists $i_{0} \geq 0$ such that $\mu^{(i)}$ is integer for all $i \geq i_{0}$.

Proof. The series $\mathcal{F}^{(i)}$ are $y_{i}$-general of order $\mathfrak{n}_{i}$, and the $\mathfrak{n}_{i}$ and the $d_{i}$ form a descending sequence of natural numbers

$$
\mathfrak{n}=\mathfrak{n}_{0} \geq d_{0} \geq \mathfrak{n}_{1} \geq d_{1} \geq \ldots
$$

By Lemma 6. $\mu^{(i)}$ fails to be integers only if $d_{i}>\mathfrak{n}_{i+1}$, and this may happen only finitely often. Hence from a certain $i_{0}$ onwards all the $\mu^{(i)}$ are integers.

By the results above, we can define $\mathfrak{p}:=\mathfrak{p}^{(0)} \cdot \ldots \cdot \mathfrak{p}^{\left(i_{0}\right)}$ such that we can write (3.14) as

$$
\beta_{0}=\beta_{0}(\varepsilon)=\sum_{h \geq h_{0}} \beta_{0}^{[h]}\left(\sigma_{0} \varepsilon\right)^{h / \mathfrak{p}},
$$

where $h_{0}=\mathfrak{h}^{(0)} \mathfrak{p}^{(1)} \cdot \ldots \cdot \mathfrak{p}^{\left(i_{0}\right)}$. By construction $\mathcal{F}^{(0)}\left(\varepsilon, \beta_{0}(\varepsilon)\right)$ vanishes to all orders, so that (3.17) is a formal solution of the implicit equation $\mathcal{F}^{(0)}\left(\varepsilon, \beta_{0}\right)=0$. We shall say that (3.17) is a Puiseux series for the plane algebroid curve defined by $\mathcal{F}^{(0)}\left(\varepsilon, \beta_{0}\right)=0$.

Lemma 8. For all $i \geq 0$ we can bound $\mathfrak{p}^{(i)} \leq \mathfrak{n}_{i}$. 
Proof. Without loss of generality we prove the result for $i=0$. By definition, there exist $k^{\prime}, j^{\prime}$ integers, with $j^{\prime} \leq \mathfrak{n}_{0}$, such that

$$
\frac{\mathfrak{h}^{(0)}}{\mathfrak{p}^{(0)}}=\mu^{(0)}=\frac{\mathfrak{r}^{(0)}-k^{\prime}}{j^{\prime}}
$$

and $\mathfrak{h}^{(0)}, \mathfrak{p}^{(0)}$ are relatively prime integers, so that $\mathfrak{p}^{(0)} \leq j^{\prime} \leq \mathfrak{n}_{0}$.

Note that by Lemma 8 we can bound $\mathfrak{p} \leq \mathfrak{n}_{0} \cdot \ldots \cdot \mathfrak{n}_{i_{0}} \leq \mathfrak{n}_{0}$ !.

Lemma 9. Let $\mathcal{F}^{(0)}\left(\varepsilon, \beta_{0}\right) \in \mathbb{R}\left\{\varepsilon, \beta_{0}\right\}$ be $\beta_{0}$-general of order $\mathfrak{n}$ and let us suppose that $\Re_{n} \neq \emptyset$ for all $n \geq 0$. Then the series (3.17), which formally solves $\mathcal{F}^{(0)}\left(\varepsilon, \beta_{0}(\varepsilon)\right) \equiv 0$, is convergent for $\varepsilon$ small enough.

Proof. Let $P_{\mathcal{F}^{(0)}}\left(\varepsilon ; \beta_{0}\right)$ be the Weierstrass polynomial [2] of $\mathcal{F}^{(0)}$ in $\mathbb{C}\{\varepsilon\}\left[\beta_{0}\right]$. If $\mathcal{F}^{(0)}$ is irreducible in $\mathbb{C}\left\{\varepsilon, \beta_{0}\right\}$, then by Theorem 1, p. 386 in [2], we have a convergent series $\beta_{0}\left(\varepsilon^{1 / \mathfrak{n}}\right)$ which solves the equation $\mathcal{F}^{(0)}\left(\varepsilon, \beta_{0}\right)=0$. Then all the following

$$
\beta_{0}\left(\varepsilon^{1 / \mathfrak{n}}\right), \beta_{0}\left(\left(e^{2 \pi i} \varepsilon\right)^{1 / \mathfrak{n}}\right), \ldots, \beta_{0}\left(\left(e^{2 \pi(\mathfrak{n}-1) i} \varepsilon\right)^{1 / \mathfrak{n}}\right)
$$

are solutions of the equation $\mathcal{F}^{(0)}\left(\varepsilon, \beta_{0}\right)=0$. Thus we have $\mathfrak{n}$ distinct roots of the Weierstrass polynomial $P_{\mathcal{F}^{(0)}}$ and they are all convergent series in $\mathbb{C}\left\{\varepsilon^{1 / \mathfrak{n}}\right\}$. But also the series (3.17) is a solution of the equation $\mathcal{F}^{(0)}\left(\varepsilon, \beta_{0}\right)=0$. Then, as a polynomial of degree $\mathfrak{n}$ has exactly $\mathfrak{n}$ (complex) roots counting multiplicity, (3.17) is one of the (3.19). In particular this means that (3.17) is convergent for $\varepsilon$ small enough.

In general, we can write

$$
\mathcal{F}^{(0)}\left(\varepsilon, \beta_{0}\right)=\prod_{i=1}^{N}\left(\mathcal{F}_{i}^{(0)}\left(\varepsilon, \beta_{0}\right)\right)^{m_{i}}
$$

for some $N \geq 1$, where the $\mathcal{F}_{i}^{(0)}$ are the irreducible factors of $\mathcal{F}^{(0)}$. Then the Puiseux series (3.17) solves one of the equations $\mathcal{F}_{i}^{(0)}\left(\varepsilon, \beta_{0}\right)=0$, and hence, by what said above, it converges for $\varepsilon$ small enough.

As a consequence of Lemma 9 we obtain the following corollary.

Theorem 1. Consider a periodic solution with frequency $\omega=p / q$ for the system (2.1). Assume that Hypotheses 1 and 圆 are satisfied with $\mathfrak{n}$ odd. Then for $\varepsilon$ small enough the system (2.1) has at least one subharmonic solution of order $q / p$. Such a solution admits a convergent power series in $|\varepsilon|^{1 / \mathfrak{n} !}$, and hence a convergent Puiseux series in $|\varepsilon|$.

Proof. If $\mathfrak{n}$ is odd, then $\Re_{n} \neq \emptyset$ for all $n \geq 0$. This trivially follows from the fact that if $\mathfrak{n}$ is odd, then there exists at least one polynomial $P_{i}^{(0)}$ associated with a segment $\mathcal{P}_{i}^{(0)}$ whose projection $\Pi_{i}^{(0)}$ on the $j$-axis is associated with a polynomial $\widetilde{P}_{i}^{(0)}$ with odd degree $\ell_{i}$. Thus such a polynomial admits a non-zero real root with odd multiplicity $\mathfrak{n}_{1}$, so that $\mathcal{F}^{(1)}\left(\varepsilon_{1}, y_{1}\right)$ is $y_{1}$-general of odd order $\mathfrak{n}_{1}$ and so on.

Hence we can apply the Newton-Puiseux process to obtain a subharmonic solution as a Puiseux series in $\varepsilon$ which is convergent for $\varepsilon$ sufficiently small by Lemma 9 .

Theorem 1 extends the results of [14. First it gives the explicit dependence of the parameter $\beta_{0}$ on $\varepsilon$, showing that it is analytic in $|\varepsilon|^{1 / \mathfrak{n} !}$. Second, it shows that it is possible to express the subharmonic solution as a convergent fractional power series in $\varepsilon$, and this allows us to push perturbation theory to arbitrarily high order.

On the other hand, the Newton-Puiseux algorithm does not allow to construct the solution within any fixed accuracy. In this regard, it is not really constructive: we know that the solution is analytic in a fractional power of $\varepsilon$, but we know neither the size of the radius of convergence nor the precision with which the solution is approximated if we stop the Newton-Puiseux at a given step. Moreover we know 
that there is at least one subharmonic solution, but we are not able to decide how many of them are possible. In fact, a subharmonic solution can be constructed for any non-zero real root of each odd-degree polynomial $P_{i}^{(n)}$ associated with each segment of $\mathcal{P}^{(n)}$ to all step of iteration, but we cannot predict $a$ priori how many possibilities will arise along the process.

However, we obtain a fully constructive algorithm if we make some further hypothesis.

Hypothesis 3 . There exists $i_{0} \geq 0$ such that at the $i_{0}$-th step of the iteration, there exists a polynomial $P^{\left(i_{0}\right)}=P^{\left(i_{0}\right)}(c)$ which has a simple root $c_{i_{0}} \in \mathbb{R}$.

Indeed, if we assume Hypothesis 3, we obtain the following result.

Theorem 2. Consider a periodic solution with frequency $\omega=p / q$ for the system (2.1). Assume that Hypotheses 1, 2] and 3 are satisfied. Then there exists an explicitly computable value $\varepsilon_{0}>0$ such that for $|\varepsilon|<\varepsilon_{0}$ the system (2.1) has at least one subharmonic solution of order $q / p$. Such a solution admits a convergent power series in $|\varepsilon|^{1 / \mathfrak{n} !}$, and hence a convergent Puiseux series in $|\varepsilon|$.

We shall see in Section 5 that, by assuming Hypothesis [3, we can use the Newton-Puiseux algorithm up to the $i_{0}$-th step (hence a finite number of times), and we can provide recursive formulae for the higher order contributions. This will allow us - as we shall see in Section [6 - to introduce a graphical representation for the subharmonic solution, and, eventually, to obtain an explicit bound on the radius of convergence of the power series expansion.

\section{Trees expansion and proof of Lemma 1}

A tree $\theta$ is defined as a partially ordered set of points $\mathfrak{v}$ (vertices) connected by oriented lines $\ell$. The lines are consistently oriented toward a unique point called the root which admits only one entering line called the root line. If a line $\ell$ connects two vertices $\mathfrak{v}_{1}, \mathfrak{v}_{2}$ and is oriented from $\mathfrak{v}_{2}$ to $\mathfrak{v}_{1}$, we say that $\mathfrak{v}_{2} \prec \mathfrak{v}_{1}$ and we shall write $\ell_{\mathfrak{v}_{2}}=\ell$. We shall say that $\ell$ exits $\mathfrak{v}_{2}$ and enters $\mathfrak{v}_{1}$. More generally we write $\mathfrak{v}_{2} \prec \mathfrak{v}_{1}$ when $\mathfrak{v}_{1}$ is on the path of lines connecting $\mathfrak{v}_{2}$ to the root: hence the orientation of the lines is opposite to the partial ordering relation $\prec$.

We denote with $V(\theta)$ and $L(\theta)$ the set of vertices and lines in $\theta$ respectively, and with $|V(\theta)|$ and $|L(\theta)|$ the number of vertices and lines respectively. Remark that one has $|V(\theta)|=|L(\theta)|$.

We consider two kinds of vertices: nodes and leaves. The leaves can only be end-points, i.e. points with no lines entering them, while the nodes can be either end-points or not. We shall not consider the tree consisting of only one leaf and the line exiting it, i.e. a tree must have at least the node which the root line exits.

We shall denote with $N(\theta)$ and $E(\theta)$ the set of nodes and leaves respectively. Here and henceforth we shall denote with $\mathfrak{v}$ and $\mathfrak{e}$ the nodes and the leaves respectively. Remark that $V(\theta)=N(\theta) \amalg E(\theta)$.

With each line $\ell=\ell_{\mathfrak{v}}$, we associate three labels $\left(h_{\ell}, \delta_{\ell}, \nu_{\ell}\right)$, with $h_{\ell} \in\{\alpha, A\}, \delta_{\ell} \in\{1,2\}$ and $\nu_{\ell} \in \mathbb{Z}$, with the constraint that $\nu_{\ell} \neq 0$ for $h_{\ell}=\alpha$ and $\delta_{\ell}=1$ for $h_{\ell}=A$. With each line $\ell=\ell_{\mathfrak{e}}$ we associate $h_{\ell}=\alpha, \delta_{\ell}=1$ and $\nu_{\ell}=0$. We shall say that $h_{\ell}, \delta_{\ell}$ and $\nu_{\ell}$ are the component label, the degree label and the momentum of the line $\ell$, respectively.

Given a node $\mathfrak{v}$, we call $r_{\mathfrak{v}}$ the number of the lines entering $\mathfrak{v}$ carrying a component label $h=\alpha$ and $s_{\mathfrak{v}}$ the number of the lines entering $\mathfrak{v}$ with component label $h=A$. We also introduce a badge label $b_{\mathfrak{v}} \in\{0,1\}$ with the constraint that $b_{\mathfrak{v}}=1$ for $h_{\ell_{\mathfrak{v}}}=\alpha$ and $\delta_{\ell_{\mathfrak{v}}}=2$, and for $h_{\ell_{\mathfrak{v}}}=A$ and $\nu_{\ell_{\mathfrak{v}}} \neq 0$, and two mode labels $\sigma_{\mathfrak{v}}, \sigma_{\mathfrak{v}}^{\prime} \in \mathbb{Z}$. We call global mode label the sum

$$
\nu_{\mathfrak{v}}=p \sigma_{\mathfrak{v}}+q \sigma_{\mathfrak{v}}^{\prime}
$$


where $q, p$ are the relatively prime integers such that $\omega\left(A_{0}\right)=p / q$, with the constraint that $\nu_{\mathfrak{v}}=0$ when $b_{\mathfrak{v}}=0$.

For all $\ell=\ell_{\mathfrak{v}}$, we set also the following conservation law

$$
\nu_{\ell}=\nu_{\ell_{\mathfrak{v}}}=\sum_{\substack{\mathfrak{w} \in N(\theta) \\ \mathfrak{w} \preceq \mathfrak{v}}} \nu_{\mathfrak{w}}
$$

i.e. the momentum of the line exiting $\mathfrak{v}$ is the sum of the momenta of the lines entering $\mathfrak{v}$ plus the global mode of the node $\mathfrak{v}$ itself.

Given a labeled tree $\theta$, where labels are defined as above, we associate with each line $\ell$ exiting a node, a propagator

$$
g_{\ell}=\left\{\begin{array}{lll}
\frac{\omega^{\prime}\left(A_{0}\right)^{\delta_{\ell}-1}}{\left(i \omega \nu_{\ell}\right)^{\delta_{\ell}}}, & h_{\ell}=\alpha, A, & \nu_{\ell} \neq 0, \\
-\frac{1}{\omega^{\prime}\left(A_{0}\right)}, & h_{\ell}=A, & \nu_{\ell}=0,
\end{array}\right.
$$

while for each line $\ell$ exiting a leaf we set $g_{\ell}=1$.

Moreover, we associate with each node $\mathfrak{v}$ a node factor

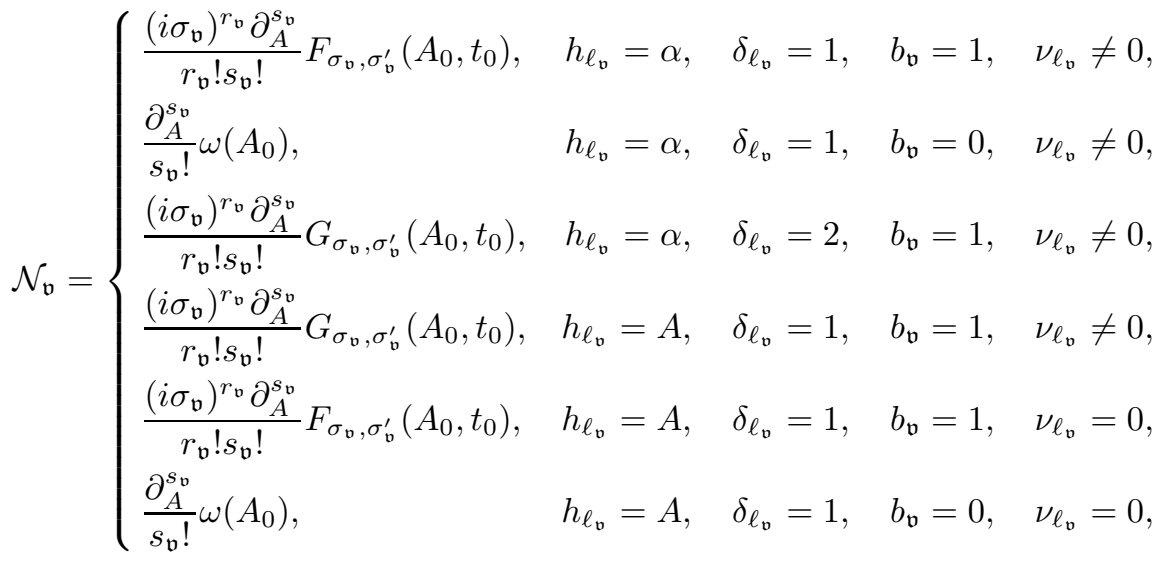

with the constraint that when $b_{\mathfrak{v}}=0$ one has $r_{\mathfrak{v}}=0$ and $s_{\mathfrak{v}} \geq 2$.

Given a labeled tree $\theta$ with propagators and node factors associated as above, we define the value of $\theta$ the number

$$
\operatorname{Val}(\theta)=\left(\prod_{\ell \in L(\theta)} g_{\ell}\right)\left(\prod_{\mathfrak{v} \in N(\theta)} \mathcal{N}_{\mathfrak{v}}\right)
$$

Remark that $\operatorname{Val}(\theta)$ is a well-defined quantity because all the propagators and node factors are bounded quantities.

For each line $\ell$ exiting a node $\mathfrak{v}$ we set $b_{\ell}=b_{\mathfrak{v}}$, while for each line $\ell$ exiting a leaf we set $b_{\ell}=0$. Given a labeled tree $\theta$, we call order of $\theta$ the number

$$
k(\theta)=\left|\left\{\ell \in L(\theta): b_{\ell}=1\right\}\right|
$$

the momentum $\nu(\theta)$ of the root line will be the total momentum, and the component label $h(\theta)$ associated to the root line will be the total component label. Moreover, we set $j(\theta)=|E(\theta)|$.

Define $\mathcal{T}_{k, \nu, h, j}$ the set of all the trees $\theta$ with order $k(\theta)=k$, total momentum $\nu(\theta)=\nu$, total component label $h(\theta)=h$ and $j(\theta)=j$ leaves. 
Lemma 10. For any tree $\theta$ labeled as before, one has $|L(\theta)|=|V(\theta)| \leq 2 k(\theta)+j(\theta)-1$.

Proof. We prove the bound $|N(\theta)| \leq 2 k(\theta)-1$ by induction on $k$.

For $k=1$ the bound is trivially satisfied, as a direct check shows: in particular, a tree $\theta$ with $k(\theta)=1$ has exactly one node and $j(\theta)$ leaves. In fact if $\theta$ has a line $\ell=\ell_{\mathfrak{v}}$ with $b_{\ell}=0$, then $\mathfrak{v}$ has $s_{\mathfrak{v}} \geq 2$ lines with component label $h=A$ entering it. Hence there are at least two lines exiting a node with $b_{\mathfrak{v}}=1$.

Assume now that the bound holds for all $k^{\prime}<k$, and let us show that then it holds also for $k$. Let $\ell_{0}$ be the root line of $\theta$ and $\mathfrak{v}_{0}$ the node which the root line exits. Call $r$ and $s$ the number of lines entering $\mathfrak{v}_{0}$ with component labels $\alpha$ and $A$ respectively, and denote with $\theta_{1}, \ldots, \theta_{r+s}$ the subtrees which have those lines as root lines. Then

$$
|N(\theta)|=1+\sum_{m=1}^{r+s}\left|N\left(\theta_{m}\right)\right| .
$$

If $\ell_{0}$ has badge label $b_{\ell_{0}}=1$ we have $|N(\theta)| \leq 1+2(k-1)-(r+s) \leq 2 k-1$, by the inductive hypothesis and by the fact that $k\left(\theta_{1}\right)+\ldots+k\left(\theta_{r+s}\right)=k-1$. If $\ell_{0}$ has badge label $b_{\ell_{0}}=0$ we have $|N(\theta)| \leq 1+2 k-(r+s) \leq 2 k-1$, by the inductive hypothesis, by the fact that $k\left(\theta_{1}\right)+\ldots+k\left(\theta_{r+s}\right)=k$, and the constraint that $s \geq 2$. Therefore the assertion is proved.

Lemma 11. The Fourier coefficients $\bar{\beta}_{\nu}^{(k, j)}, \nu \neq 0$, and $\bar{B}_{\nu}^{(k, j)}$ can be written in terms of trees as

$$
\begin{aligned}
& \bar{\beta}_{\nu}^{(k, j)}=\sum_{\theta \in \mathcal{T}_{k, \nu, \alpha, j}} \operatorname{Val}(\theta), \quad \nu \neq 0, \\
& \bar{B}_{\nu}^{(k, j)}=\sum_{\theta \in \mathcal{T}_{k, \nu, A, j}} \operatorname{Val}(\theta), \quad \nu \in \mathbb{Z},
\end{aligned}
$$

for all $k \geq 1, j \geq 0$.

Proof. First we consider trees without leaves, i.e. the coefficients $\bar{\beta}_{\nu}^{(k, 0)}, \nu \neq 0$, and $\bar{B}_{\nu}^{(k, 0)}$. For $k=1$ is a direct check. Now let us suppose that the assertion holds for all $k<\bar{k}$. Let us write $f_{\alpha}=\bar{\beta}, f_{A}=\bar{B}$ and represent the coefficients $f_{\nu, h}^{(k, 0)}$ with the graph elements in Figure 2, as a line with label $\nu$ and $h=\alpha, A$ respectively, exiting a ball with label $(k, 0)$.

$$
\begin{aligned}
& \bar{\beta}_{\nu}^{(k, 0)}=\overbrace{\alpha 1 \nu}^{(k, 0)}+\frac{1}{\alpha 2} \nu^{(k, 0)} \\
& \bar{B}_{\nu}^{(k, 0)}=\overbrace{A \dot{1} \nu}^{(k, 0)}
\end{aligned}
$$

Figure 2: Graph elements.

Then we can represent each equation of (2.21) graphically as in Figure 3, simply representing each factor $f_{h_{i}, \nu_{i}}^{\left(k_{i}, 0\right)}$ in the r.h.s. as a graph element according to Figure 2 the lines of all such graph elements enter the same node $\mathfrak{v}_{0}$. 


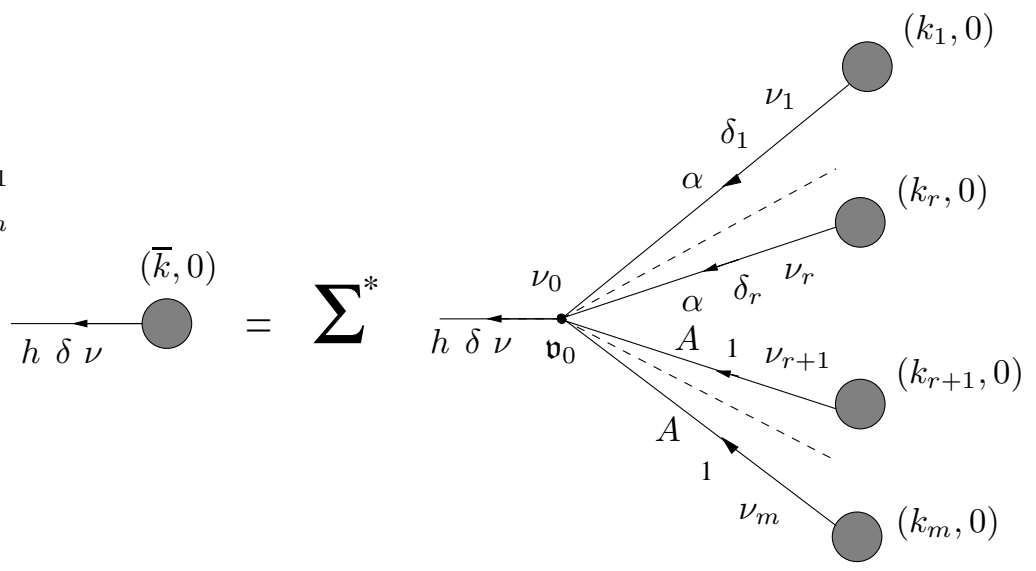

Figure 3: Graphical representation for the recursive equations (2.23).

The root line $\ell_{0}$ of such trees will carry a component label $h=\alpha, A$ for $f=\bar{\beta}, \bar{B}$ respectively, and a momentum label $\nu$. Hence, by inductive hypothesis, one obtains

$$
\begin{aligned}
f_{h, \nu}^{(\bar{k}, 0)} & =\sum^{*} g_{\ell_{0}} \mathcal{N}_{\mathfrak{v}_{0}} f_{h_{1}, \nu_{1}}^{\left(k_{1}, 0\right)} \ldots f_{h_{m}, \nu_{m}}^{\left(k_{m}, 0\right)} \\
& =\sum^{*} g_{\ell_{0}} \mathcal{N}_{\mathfrak{v}_{0}}\left(\sum_{\theta \in \mathcal{T}_{\kappa_{1}, \nu_{1}, h_{1}, 0}} \operatorname{Val}(\theta)\right) \ldots\left(\sum_{\theta \in \mathcal{T}_{\kappa_{m}, \nu_{m}, h_{m}, 0}} \operatorname{Val}(\theta)\right)=\sum_{\theta \in \mathcal{T}_{\bar{k}, \nu, h, 0}} \operatorname{Val}(\theta),
\end{aligned}
$$

where $m=r_{0}+s_{0}$, and we write $\sum^{*}$ for the sum over all the labels admitted by the constraints, so that the assertion is proved for all $k$ and for $j=0$.

Now we consider $k$ as fixed and we prove the statement by induction on $j$. The case $j=0$ has already been discussed. Finally we assume that the assertion holds for $j=j^{\prime}$ and show that then it holds for $j^{\prime}+1$. Notice that a tree $\theta \in \mathcal{T}_{k, \nu, h, j^{\prime}+1}$, for both $h=\alpha, A$ can be obtained by considering a suitable tree $\theta_{0} \in \mathcal{T}_{k, \nu, h, j^{\prime}}$ attaching an extra leaf to a node of $\theta_{0}$ and applying an extra derivative $\partial_{\alpha}$ to the node factor associated with that node. If one considers all the trees that can be obtained in such a way from the same $\theta_{0}$ and sums together all those contributions, one finds a quantity proportional to $\partial_{\alpha} \operatorname{Val}\left(\theta_{0}\right)$. Then if we sum over all possible choices of $\theta_{0}$, we reconstruct $\bar{\beta}_{\nu}^{\left(k, j^{\prime}+1\right)}$ for $h=\alpha$ and $\bar{B}_{\nu}^{\left(k, j^{\prime}+1\right)}$ for $h=A$. Hence the assertion follows.

Proposition 1. The formal solution (2.19) of the system (2.10), given by the recursive equations (2.21), converges for $\varepsilon$ and $\beta_{0}$ small enough.

Proof. First of all we remark that by Lemma 10, the number of unlabeled trees of order $k$ and $j$ leaves is bounded by $4^{2 k+j} \times 2^{2 k+j}=8^{2 k+j}$. The sum over all labels except the mode labels and the momenta is bounded again by a constant to the power $k$ times a constant to the power $j$, simply because all such labels can assume only a finite number of values. Now by the analyticity assumption on the functions $F$ and $G$, we have the bound

$$
\begin{aligned}
& \left|\frac{\left(i \sigma_{0}\right)^{r}}{r !} \frac{\partial_{A}^{s}}{s !} F_{\sigma_{0}, \sigma_{0}^{\prime}}\left(A_{0}, t_{0}\right)\right| \leq \mathcal{Q} R^{r} S^{s} e^{-\kappa\left(\left|\sigma_{0}\right|+\left|\sigma_{0}^{\prime}\right|\right),} \\
& \left|\frac{\left(i \sigma_{0}\right)^{r}}{r !} \frac{\partial_{A}^{s}}{s !} G_{\sigma_{0}, \sigma_{0}^{\prime}}\left(A_{0}, t_{0}\right)\right| \leq \mathcal{Q} R^{r} S^{s} e^{-\kappa\left(\left|\sigma_{0}\right|+\left|\sigma_{0}^{\prime}\right|\right)},
\end{aligned}
$$


for suitable positive constants $\mathcal{Q}, R, S, \kappa$, and we can imagine, without loss of generality, that $\mathcal{Q}$ and $S$ are such that $\left|\partial_{A}^{s} \omega\left(A_{0}\right) / s !\right| \leq \mathcal{Q} S^{s}$. This gives us a bound for the node factors. The propagators can be bounded by

$$
\left|g_{\ell}\right| \leq \max \left\{\left|\frac{\omega^{\prime}\left(A_{0}\right)}{\omega^{2}}\right|,\left|\frac{1}{\omega^{\prime}\left(A_{0}\right)}\right|,\left|\frac{1}{\omega}\right|, 1\right\},
$$

so that the product over all the lines can be bounded again by a constant to the power $k$ times a constant to the power $j$.

Thus the sum over the mode labels - which uniquely determine the momenta - can be performed by using for each node half the exponential decay factor provided by (4.10). Then we obtain

$$
\left|\bar{\beta}_{\nu}^{(k, j)}\right| \leq C_{1} C_{2}^{k} C_{3}^{j} e^{-\kappa|\nu| / 2}, \quad\left|\bar{B}_{\nu}^{(k, j)}\right| \leq C_{1} C_{2}^{k} C_{3}^{j} e^{-\kappa|\nu| / 2},
$$

for suitable constants $C_{1}, C_{2}$ and $C_{3}$. This provides the convergence of the series (2.19) for $|\varepsilon|<C_{2}^{-1}$ and $\left|\beta_{0}\right|<C_{3}^{-1}$.

\section{Formal solubility of the equations of motion}

Assume that Hypotheses 1 , 2 and 3 are satisfied. Let us set $\eta:=|\varepsilon|^{1 / \mathfrak{p}}$, where $\mathfrak{p}=\mathfrak{p}^{(0)} \cdot \ldots \cdot \mathfrak{p}^{\left(i_{0}\right)}$. We search for a formal solution $(\alpha(t), A(t))$ of (2.1), with $\alpha(t)=\alpha_{0}(t)+\beta_{0}+\widetilde{\beta}(t)$ and $A(t)=A_{0}+B(t)$, where

$$
\beta_{0}=\sum_{k \geq 1} \eta^{k} \beta_{0}^{[k]}, \quad \widetilde{\beta}(t)=\sum_{\substack{\nu \in \mathbb{Z} \\ \nu \neq 0}} e^{i \nu \omega t} \sum_{k \geq 1} \eta^{k} \widetilde{\beta}_{\nu}^{[k]}, \quad B(t)=\sum_{\nu \in \mathbb{Z}} e^{i \nu \omega t} \sum_{k \geq 1} \eta^{k} B_{\nu}^{[k]},
$$

and the coefficients $\beta_{0}^{[k]}, \widetilde{\beta}_{\nu}^{[k]}$ and $B_{\nu}^{[k]}$ solve

$$
\left\{\begin{array}{l}
\widetilde{\beta}_{\nu}^{[k]}=\frac{\Phi_{\nu}^{[k]}}{i \omega \nu}+\omega^{\prime}\left(A_{0}\right) \frac{\Gamma_{\nu}^{[k]}}{(i \omega \nu)^{2}}, \quad B_{\nu}^{[k]}=\frac{\Gamma_{\nu}^{[k]}}{i \omega \nu}, \quad \nu \neq 0, \\
B_{0}^{[k]}=-\frac{\Phi_{0}^{[k]}}{\omega^{\prime}\left(A_{0}\right)}, \quad \Gamma_{0}^{[k]}=0,
\end{array}\right.
$$

with the functions $\Gamma_{\nu}^{[k]}$ and $\Phi_{\nu}^{[k]}$ recursively defined as

$$
\begin{aligned}
\Gamma_{\nu}^{[k]} & =\sum_{m \geq 0} \sum_{r+s=m} \sum_{\substack{p \\
p \sigma_{0}+q \sigma_{0}^{\prime}+\nu_{1}+\ldots+\nu_{m}=\nu \\
k_{1}+\ldots+k_{m}=k-\mathfrak{p}}} \frac{\left(i \sigma_{0}\right)^{r}}{r !} \frac{\partial_{A}^{s}}{s !} G_{\sigma_{0}, \sigma_{0}^{\prime}}\left(A_{0}, t_{0}\right) \beta_{\nu_{1}}^{\left[k_{1}\right]} \cdots \beta_{\nu_{r}}^{\left[k_{r}\right]} B_{\nu_{r+1}}^{\left[k_{r+1}\right]} \cdots B_{\nu_{m}}^{\left[k_{m}\right]}, \\
\Phi_{\nu}^{[k]} & =\sum_{m \geq 0} \sum_{r+s=m} \sum_{\substack{\left.r+\sigma_{0}\right)^{r} \\
p \sigma_{0}+q \sigma_{0}^{\prime}+\nu_{1}+\ldots+\nu_{m}=\nu \\
k_{1}+\ldots+k_{m}=k-\mathfrak{p}}} \frac{\partial_{A}^{s}}{s !} F_{\sigma_{0}, \sigma_{0}^{\prime}}\left(A_{0}, t_{0}\right) \beta_{\nu_{1}}^{\left[k_{1}\right]} \cdots \beta_{\nu_{r}}^{\left[k_{r}\right]} B_{\nu_{r+1}}^{\left[k_{r+1}\right]} \cdots B_{\nu_{m}}^{\left[k_{m}\right]}, \\
& +\sum_{s \geq 2} \sum_{\substack{\nu_{1}+\ldots+\nu_{s}=\nu \\
k_{1}+\ldots+k_{s}=k}} \frac{\partial_{A}^{s}}{s !} \omega\left(A_{0}\right) B_{\nu_{1}}^{\left[k_{1}\right]} \ldots B_{\nu_{s}}^{\left[k_{s}\right]},
\end{aligned}
$$

where $\beta_{\nu}^{[k]}=\widetilde{\beta}_{\nu}^{[k]}$ for $\nu \neq 0$. We use a different notation for the Taylor coefficients to stress that we are expanding in $\eta$.

We say that the integral equations (2.9), and hence the equations (5.2), are satisfied up to order $\bar{k}$ if there exists a choice of the parameters $\beta_{0}^{[1]}, \ldots \beta_{0}^{[\bar{k}]}$ which make the relations (5.2) to be satisfied for all $k=1, \ldots, \bar{k}$. 
Lemma 12. The equations (5.2) are satisfied up to order $k=\mathfrak{p}-1$ with $\widetilde{\beta}_{\nu}^{[k]}$ and $B_{\nu}^{[k]}$ identically zero for all $k=1, \ldots, \mathfrak{p}-1$ and for any choice of the constants $\beta_{0}^{[1]}, \ldots \beta_{0}^{[\mathfrak{p}-1]}$.

Proof. One has $\varepsilon=\sigma \eta^{\mathfrak{p}}$, with $\sigma=\operatorname{sign}(\varepsilon)$, so that $\Phi_{\nu}^{[k]}=\Gamma_{\nu}^{[k]}=0$ for all $k<\mathfrak{p}$ and all $\nu \in \mathbb{Z}$, independently of the values of the constants $\beta_{0}^{[1]}, \ldots \beta_{0}^{[\mathfrak{p}-1]}$. Moreover $\widetilde{\beta}_{\nu}^{[k]}=B_{\nu}^{[k]}=0$ for all $k<\mathfrak{p}$.

Lemma 13. The equations (5.2) are satisfied up to order $k=\mathfrak{p}$, for any choice of the constants $\beta_{0}^{[1]}, \ldots \beta_{0}^{[\mathfrak{p}]}$.

Proof. One has $\Gamma^{[\mathfrak{p}]}=G\left(\alpha_{0}(t), A_{0}, t+t_{0}\right)$ and $\Phi^{[\mathfrak{p}]}=F\left(\alpha_{0}(t), A_{0}, t+t_{0}\right)$, so that

$$
\Gamma_{\nu}^{[\mathfrak{p}]}=\sum_{p \sigma_{0}+q \sigma_{0}^{\prime}=\nu} G_{\sigma_{0}, \sigma_{0}^{\prime}}\left(A_{0}\right), \quad \Phi_{\nu}^{[\mathfrak{p}]}=\sum_{p \sigma_{0}+q \sigma_{0}^{\prime}=\nu} F_{\sigma_{0}, \sigma_{0}^{\prime}}\left(A_{0}\right)
$$

Thus, $\widetilde{\beta}_{\nu}^{[\mathfrak{p}]}$ and $B_{\nu}^{[\mathfrak{p}]}$ can be obtained from (5.2). Finally $\Gamma_{0}^{[\mathfrak{p}]}=M\left(t_{0}\right)$ by definition, and one has $M\left(t_{0}\right)=0$ by Hypothesis 2 Hence also the last equation of (5.2) is satisfied.

Let us set

$$
\begin{array}{ll}
\mathfrak{h}_{0}=\mathfrak{h}^{(0)} \mathfrak{p}^{(1)} \cdot \ldots \cdot \mathfrak{p}^{\left(i_{0}\right)}, & \mathfrak{s}_{0}=\mathfrak{s}^{(0)} \mathfrak{p}^{(1)} \cdot \ldots \cdot \mathfrak{p}^{\left(i_{0}\right)} \\
\mathfrak{h}_{1}=\mathfrak{h}_{0}+\mathfrak{h}^{(1)} \mathfrak{p}^{(2)} \cdot \ldots \cdot \mathfrak{p}^{\left(i_{0}\right)}, & \mathfrak{s}_{1}=\mathfrak{s}_{0}+\mathfrak{s}^{(1)} \mathfrak{p}^{(2)} \cdot \ldots \cdot \mathfrak{p}^{\left(i_{0}\right)}, \\
\mathfrak{h}_{2}=\mathfrak{h}_{1}+\mathfrak{h}^{(2)} \mathfrak{p}^{(3)} \cdot \ldots \cdot \mathfrak{p}^{\left(i_{0}\right)}, & \mathfrak{s}_{2}=\mathfrak{s}_{1}+\mathfrak{s}^{(2)} \mathfrak{p}^{(3)} \cdot \ldots \cdot \mathfrak{p}^{\left(i_{0}\right)} \\
\vdots & \vdots \\
\mathfrak{h}_{i_{0}}=\mathfrak{h}_{i_{0}-1}+\mathfrak{h}^{\left(i_{0}\right)}, & \mathfrak{s}_{i_{0}}=\mathfrak{s}_{i_{0}-1}+\mathfrak{s}^{\left(i_{0}\right)}
\end{array}
$$

Lemma 14. The equations (5.2) are satisfied up to order $k=\mathfrak{p}+\mathfrak{s}_{i_{0}}$ provided $\beta_{0}^{\left[\mathfrak{h}_{i}\right]}=c_{i}$, with $c_{i}$ the real root of a polynomial $P^{(i)}(c)$ of the $i$-th step of iteration step of the Newton-Puiseux process, for $i=0, \ldots, i_{0}$, and $\beta_{0}^{\left[k^{\prime}\right]}=0$ for all $k^{\prime} \leq \mathfrak{h}_{i_{0}}, k^{\prime} \neq \mathfrak{h}_{i}$ for any $i$.

Proof. If $\beta_{0}^{\left[k^{\prime}\right]}=0$ for all $1<k^{\prime}<\mathfrak{h}_{0}$, one has $\Gamma_{0}^{[k]}=0$ for all $\mathfrak{p}<k<\mathfrak{p}+\mathfrak{s}_{0}$, while $\Gamma_{0}^{\left[\mathfrak{p}+\mathfrak{s}_{0}\right]}=P^{(0)}\left(\beta_{0}^{\left[\mathfrak{h}_{0}\right]}\right)$, so that $\Gamma_{0}^{\left[\mathfrak{p}+\mathfrak{s}_{0}\right]}=0$ for $\beta_{0}^{\left[\mathfrak{h}_{0}\right]}=c_{0}$. Thus $\Gamma_{0}^{[k]}=0$ for $\mathfrak{p}+\mathfrak{s}_{0}<k<\mathfrak{p}+\mathfrak{s}_{1}$ provided $\beta_{0}^{\left[k^{\prime}\right]}=0$ for all $\mathfrak{h}_{0}<k^{\prime}<\mathfrak{h}_{1}$, while $\Gamma_{0}^{\left[\mathfrak{p}+\mathfrak{s}_{1}\right]}=P^{(1)}\left(\beta_{0}^{\left[\mathfrak{h}_{1}\right]}\right)$, so that $\Gamma_{0}^{\left[\mathfrak{p}+\mathfrak{s}_{1}\right]}=0$ for $\beta_{0}^{\left[\mathfrak{h}_{1}\right]}=c_{1}$, and so on.

Hence if we set $\beta_{0}^{\left[\mathfrak{h}_{i}\right]}=c_{i}$, for all $i=0, \ldots, i_{0}$, and $\beta_{0}^{\left[k^{\prime}\right]}=0$ for all $k^{\prime}<\mathfrak{h}_{i_{0}}, k^{\prime} \neq \mathfrak{h}_{i}$ for any $i=0, \ldots, i_{0}$, one has $\Gamma_{0}^{[k]}=0$ for all $\mathfrak{p}<k \leq \mathfrak{p}+\mathfrak{s}_{i_{0}}$. Moreover, $\Phi_{\nu}^{[k]}$ and $\Gamma_{\nu}^{[k]}$ are well-defined for such values of $k$. Hence (5.2) can be solved up to order $k=\mathfrak{p}+\mathfrak{s}_{i_{0}}$, indipendently of the values of the constants $\beta_{0}^{\left[k^{\prime}\right]}$ for $k^{\prime}>\mathfrak{h}_{i_{0}}$.

By Lemma 14 we can write

$$
\beta_{0}=\beta_{0}(\eta):=c_{0} \eta^{\mathfrak{h}_{0}}+c_{1} \eta^{\mathfrak{h}_{1}}+\ldots+c_{i_{0}} \eta^{\mathfrak{h}_{i_{0}}}+\sum_{k \geq 1} \beta_{0}^{\left[\mathfrak{h}_{i_{0}}+k\right]} \eta^{\mathfrak{h}_{i_{0}}+k}
$$

Lemma 15. The equations (5.2) are satisfied up to any order $k=\mathfrak{p}+\mathfrak{s}_{i_{0}}+\kappa, \kappa \geq 1$ provided the constants $\beta_{0}^{\left[\mathfrak{h}_{i_{0}}+\kappa^{\prime}\right]}$ are suitably fixed up to order $\kappa^{\prime}=\kappa$. 
Proof. By substituting (5.6) and $\varepsilon=\sigma \eta^{\mathfrak{p}}$, with $\sigma=\operatorname{sign}(\varepsilon)$, in $\Gamma_{0}\left(\varepsilon, \beta_{0}\right)$ we obtain

$$
\begin{aligned}
\Gamma_{0}\left(\sigma \eta^{\mathfrak{p}}, \beta_{0}(\eta)\right) & =\sigma \eta^{\mathfrak{p}} \sum_{s_{1}, j \geq 0} Q_{s_{1}, j} \eta^{s_{1} \mathfrak{p}} \sum_{\substack{m_{0}+\ldots+m_{i_{0}}+m=j \\
m, m_{i} \geq 0}} J\left(j, m_{0}, \ldots, m_{i_{0}}, m\right) \times \\
& \times \eta^{m_{0} \mathfrak{h}_{0}+\ldots+m_{i_{0}} \mathfrak{h}_{i_{0}}} c_{0}^{m_{0}} \ldots \cdot c_{i_{0}}^{m_{i_{0}}} \sum_{n \geq 0} \eta^{m \mathfrak{h}_{i_{0}}+n} \sum_{\substack{n_{1}+\ldots+n_{m}=n \\
n_{i} \geq 1}} \beta_{0}^{\left[\mathfrak{h}_{i_{0}}+n_{1}\right]} \ldots \beta_{0}^{\left[\mathfrak{h}_{i_{0}}+n_{m}\right]}
\end{aligned}
$$

where $Q_{s_{1}, j}=\mathcal{F}_{s_{1}, j}^{(0)} \sigma^{s_{1}}$ and

$$
J\left(j, m_{0}, \ldots, m_{i_{0}}, m\right):=\frac{j !}{m_{0} ! \ldots m_{i_{0}} ! m !} .
$$

For any $\kappa \geq 1$ one has, by rearranging the sums,

$$
\sigma \Gamma_{0}^{\left[\mathfrak{p}+\mathfrak{s}_{i_{0}}+\kappa\right]}=\sum_{\begin{array}{c}
m, m_{i}, n, s_{1}, j \geq 0 \\
m_{0}+\ldots+m_{i}+m=j \\
s_{1} \mathfrak{p}+m_{0} \mathfrak{h}_{0}+\ldots+m_{i_{0}} \mathfrak{h}_{i_{0}}+m \mathfrak{h}_{i_{0}}=\mathfrak{s}_{i_{0}}+n
\end{array}} J\left(j, m_{0}, \ldots, m_{i_{0}}, m\right) Q_{s_{1}, j} c_{0}^{m_{0}} \ldots c_{\substack{i_{0} \\
n_{1}+\ldots+n_{m}}}^{m_{i_{0}}} \sum_{n_{i} \geq 1}^{\left[\mathfrak{h}_{i_{0}}+n_{1}\right]} \ldots \beta_{0}^{\left[\mathfrak{h}_{i_{0}}+n_{m}\right]},
$$

so that the last equation of (5.2) gives for $\kappa \geq 1$

$$
\begin{aligned}
& \sum_{m_{i}, s_{1}, j \geq 0} m_{i_{0}} J\left(j, m_{0}, \ldots, m_{i_{0}}, m\right) Q_{s_{1}, j} c_{0}^{m_{0}} \ldots c_{i_{0}-1}^{m_{i_{0}}-1} c_{i_{0}}^{m_{i_{0}}-1} \beta_{0}^{\left[\mathfrak{h}_{i_{0}}+\kappa\right]} \\
& m_{0}+\ldots+m_{i_{0}}+1=j \\
& s_{1} \mathfrak{p}+m_{0} \mathfrak{h}_{0}+\ldots+m_{i_{0}} \mathfrak{h}_{i_{0}}=\mathfrak{s}_{i_{0}}
\end{aligned}
$$

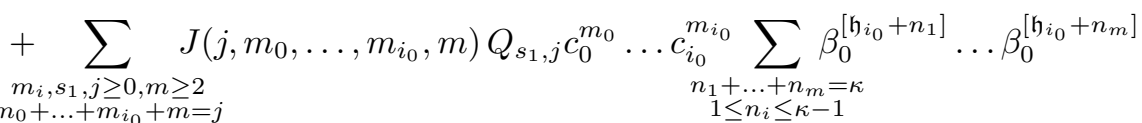

$$
\begin{aligned}
& \begin{array}{c}
m_{0}+\ldots+m_{i_{0}}+m=j \\
s_{1} \mathfrak{p}+m_{0} \mathfrak{h}_{0}+\ldots+m_{i_{0}} \mathfrak{h}_{i_{0}}+m \mathfrak{h}_{i_{0}}=\mathfrak{s}_{i_{0}}
\end{array} \\
& +\sum_{\substack{m, m_{i}, s_{1}, j \geq 0, n \geq 1 \\
m_{0}+\ldots+m_{i_{0}}+m=j}} J\left(j, m_{0}, \ldots, m_{i_{0}}, m\right) Q_{s_{1}, j} c_{0}^{m_{0}} \ldots c_{\substack{i_{0} \\
n_{1}+\ldots+n_{m}}}^{m_{i_{0}}} \sum_{\substack{n_{i} \geq 1 \\
n_{i}-n}} \beta_{0}^{\left[\mathfrak{h}_{i_{0}}+n_{1}\right]} \ldots \beta_{0}^{\left[\mathfrak{h}_{i_{0}}+n_{m}\right]}=0 \\
& s_{1} \mathfrak{p}+m_{0} \mathfrak{h}_{0}+\ldots+m_{i_{0}} \mathfrak{h}_{i_{0}}+m \mathfrak{h}_{i_{0}}=\mathfrak{s}_{i_{0}}+n
\end{aligned}
$$

where all terms but those in the first line contain only coefficients $\beta_{0}^{\left[\mathfrak{h}_{0}+\kappa^{\prime}\right]}$ with $\kappa^{\prime}<\kappa$.

Recall that by Hypothesis 3

$$
\sum_{\substack{s_{1}, j \geq 0 \\ m_{0}+\ldots+m_{i_{0}}=j \\ s_{1} \mathfrak{p}+m_{0} \mathfrak{h}_{0}+\ldots+m_{i_{0}} \mathfrak{h}_{i_{0}}=\mathfrak{s}_{i_{0}}}} m_{i_{0}} J\left(j, m_{0}, \ldots, m_{i_{0}}, m\right) Q_{s_{1}, j} c_{0}^{m_{0}} \ldots c_{i_{0}-1}^{m_{i_{0}}-1} c_{i_{0}}^{m_{i_{0}}-1}=\frac{\mathrm{d} P^{\left(i_{0}\right)}}{\mathrm{d} c}\left(c_{i_{0}}\right)=: C \neq 0,
$$

so that we can use (5.10) to express $\beta_{0}^{\left[\mathfrak{h}_{i_{0}}+\kappa\right]}$ in terms of the coefficients $\beta_{0}^{\left[\mathfrak{h}_{i_{0}}+\kappa^{\prime}\right]}$ of lower orders $\kappa^{\prime}<\kappa$. Thus we can conclude that the equations (5.2) are satisfied up to order $k$ provided the coefficients $\beta_{0}^{\left[\mathfrak{h}_{i_{0}}+\kappa^{\prime}\right]}$ are fixed as

$$
\beta_{0}^{\left[\mathfrak{h}_{i}+\kappa^{\prime}\right]}=-\frac{1}{C} \widetilde{G}^{\left[\kappa^{\prime}\right]}\left(c_{0}, \ldots, c_{i_{0}}, \beta_{0}^{\left[\mathfrak{h}_{i}+1\right]}, \ldots, \beta_{0}^{\left[\mathfrak{h}_{i 0}+\kappa^{\prime}-1\right]}\right),
$$

for all $1 \leq \kappa^{\prime} \leq \kappa$, where $\widetilde{G}^{[\kappa]}\left(c_{0}, \ldots, c_{i_{0}}, \beta_{0}^{\left[\mathfrak{h}_{i_{0}}+1\right]}, \ldots, \beta_{0}^{\left[\mathfrak{h}_{i_{0}}+\kappa-1\right]}\right)$ is given by the sum of the second and third lines in (5.10).

We can summarise the results above into the following statement.

Proposition 2. The equations (5.2) are satisfied to any order $k$ provided the constants $\beta_{0}^{[k]}$ are suitably fixed. In particular $\widetilde{\beta}_{\nu}^{[k]}=B_{\nu}^{[k]}=B_{0}^{[k]}=0$ for $k<\mathfrak{p}$ and $\beta_{0}^{[k]}=0$ for $k<\mathfrak{h}_{i_{0}}, k \neq \mathfrak{h}_{i}$ for any $i=0, \ldots, i_{0}$. 


\section{Diagrammatic rules for the Puiseux series}

In order to give a graphical representation of the coefficients $\beta_{0}^{[k]}, \widetilde{\beta}_{\nu}^{[k]}$ and $B_{\nu}^{[k]}$ in (5.1), we shall consider a different tree expansion with respect to that of Section 4 . We shall perform an iterative construction, similar to the one performed through the proof of Lemma 11, starting from equations (5.2) for the coefficients $\widetilde{\beta}_{\nu}^{[k]}, B_{\nu}^{[k]}$ for $k \geq \mathfrak{p}$, and from (5.12) for $\beta_{0}^{[k]}, k \geq \mathfrak{h}_{i_{0}}+1$.

Let us consider a tree with leaves. We associate with each leaf $\mathfrak{e}$ a leaf label $\mathfrak{a}_{\mathfrak{e}}=0, \ldots, i_{0}$.

For $k=\mathfrak{p}$ we represent the coefficients $\widetilde{\beta}_{\nu}^{[\mathfrak{p}]}$ and $B_{\nu}^{[\mathfrak{p}]}$ as a line exiting a node, while for $k=\mathfrak{h}_{i}$, $i=0, \ldots, i_{0}$ we represent $\beta_{0}^{\left[\mathfrak{h}_{i}\right]}$ as a line exiting a leaf with leaf label $\mathfrak{a}_{i}$.

Now we represent each coefficient as a graph element according to Figure 4, as a line exiting a ball with order label $k$, with $k \geq \mathfrak{h}_{i_{0}}+1$ for the coefficients $\beta_{0}^{[k]}$, and $k \geq \mathfrak{p}+1$ for the coefficients $\widetilde{\beta}_{\nu}^{[k]}$ and $B_{\nu}^{[k]}$; we associate with the line a component label $h_{\ell} \in\left\{\beta_{0}, \widetilde{\beta}, B\right\}$, a degree label $\delta_{\ell} \in\{1,2\}$ with the constraint that $\delta_{\ell}=1$ for $h_{\ell}=B, \beta_{0}$, and momentum label $\nu_{\ell} \in \mathbb{Z}$, with the constraint that $\nu_{\ell} \neq 0$ for $h_{\ell}=\widetilde{\beta}$, while $\nu_{\ell}=0$ for $h_{\ell}=\beta_{0}$.

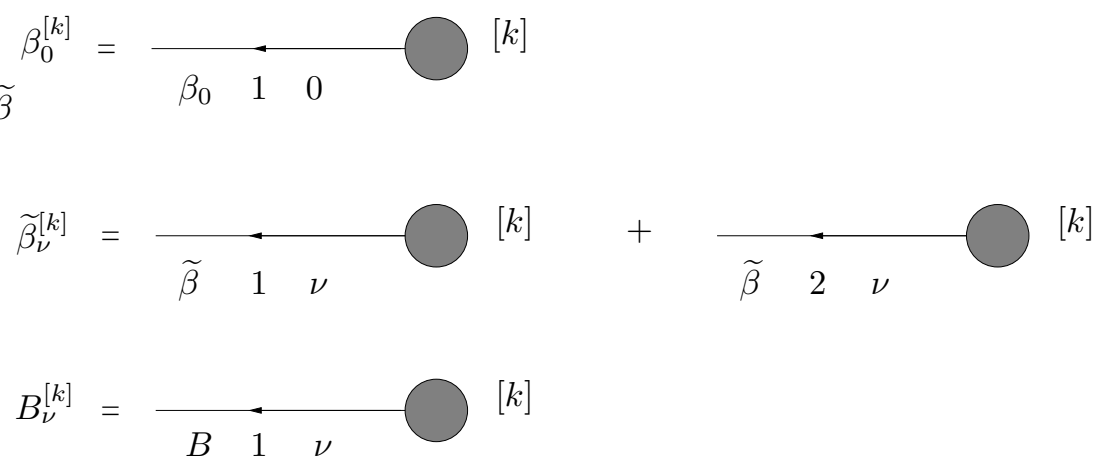

Figure 4: Graph elements.

Hence we can represent the first three equations in (5.2) graphically, representing each factor $\beta_{\nu_{i}}^{\left[k_{i}\right]}$ and $B_{\nu_{i}}^{\left[k_{i}\right]}$ in (5.3) as graph elements: again the lines of such graph elements enter the same node $\mathfrak{v}_{0}$.

We associate with $\mathfrak{v}_{0}$ a badge label $b_{\mathfrak{v}_{0}} \in\{0,1\}$ by setting $b_{\mathfrak{v}_{0}}=1$ for $h_{\ell_{0}}=\widetilde{\beta}$ and $\delta_{\ell_{0}}=2$, and for $h_{\ell_{0}}=B$ and $\nu_{\ell_{0}} \neq 0$. We call $r_{\mathfrak{v}_{0}}$ the number of the lines entering $\mathfrak{v}_{0}$ with component label $h=\beta_{0}, \widetilde{\beta}$, and $s_{\mathfrak{v}_{0}}$ the number of the lines entering $\mathfrak{v}_{0}$ with component label $h=B$, with the constraint that if $b_{\mathfrak{v}_{0}}=0$ one has $r_{\mathfrak{v}_{0}}=0$ and $s_{\mathfrak{v}_{0}} \geq 2$. Finally we associate with $\mathfrak{v}_{0}$ two mode labels $\sigma_{\mathfrak{v}_{0}}, \sigma_{\mathfrak{v}_{0}^{\prime}} \in \mathbb{Z}$ and the global mode label $\nu_{\mathfrak{v}_{0}}$ defined as in (4.1), and we impose the conservation law

$$
\nu_{\ell_{\mathfrak{v}_{0}}}=\nu_{\mathfrak{v}_{0}}+\sum_{i=1}^{r_{\mathfrak{v}_{0}}+s_{\mathfrak{v}_{0}}} \nu_{\ell_{i}}
$$

where $\ell_{1}, \ldots, \ell_{r_{\mathfrak{v}_{0}}+s_{\mathfrak{v}_{0}}}$ are the lines entering $\mathfrak{v}_{0}$. 
We also force the following conditions on the order labels

$$
\begin{aligned}
\sum_{i=1}^{r_{\mathfrak{v}_{0}}+s_{\mathfrak{v}_{0}}} k_{i}=k-\mathfrak{p}, & b_{\mathfrak{v}_{0}}=1, \\
\sum_{i=1}^{s_{\mathfrak{v}_{0}}} k_{i}=k, & b_{\mathfrak{v}_{0}}=0,
\end{aligned}
$$

which reflect the condition on the sums in (5.3).

Finally we associate with $\mathfrak{v}=\mathfrak{v}_{0}$ a node factor $\mathcal{N}_{\mathfrak{v}}^{*}=\sigma^{b_{\mathfrak{v}}} \mathcal{N}_{\mathfrak{v}}$, with $\sigma=\operatorname{sign}(\varepsilon)$ and $\mathcal{N}_{\mathfrak{v}}$ defined as in (4.4), and with the line $\ell=\ell_{\mathfrak{v}_{0}}$ a propagator $g_{\ell}^{*}=g_{\ell}$, with $g_{\ell}$ defined as in (4.3). The only difference with respect to Section 4 is that the component label can assume the values $\widetilde{\beta}, B$, which have the rôle of $\alpha, A$ respectively.

The coefficients $\beta_{0}^{\left[\mathfrak{h}_{i}+\kappa\right]}, \kappa \geq 1$, have to be treated in a different way.

First of all we point out that also the coefficients $\bar{\Gamma}_{0}^{(k, j)}$ in (2.20) can be represented in terms of sum of trees with leaves as in Section 4. In fact we can repeat the iterative construction of Lemma 11, simply by defining $\mathcal{T}_{k, 0, \Gamma, j}$ as the set of the trees contributing to $\bar{\Gamma}_{0}^{(k, j)}$, setting $g_{\ell_{0}}=1, h_{\ell_{0}}=\Gamma, \delta_{\ell_{0}}=1, \nu_{\ell_{0}}=0$, $b_{\mathfrak{v}_{0}}=1$ and

$$
\mathcal{N}_{\mathfrak{v}_{0}}=\frac{\left(i \sigma_{\mathfrak{v}_{0}}\right)^{r_{\mathfrak{v}_{0}}} \partial_{A}^{s_{\mathfrak{v}_{0}}}}{r_{\mathfrak{v}_{0}} ! s_{\mathfrak{v}_{0}} !} G_{\sigma_{\mathfrak{v}_{0}}, \sigma_{\mathfrak{v}_{0}}^{\prime}}\left(A_{0}, t_{0}\right)
$$

and no further difficulties arise.

Recall that the coefficients $Q_{s_{1}, j}$ in (5.10) are defined as $Q_{s_{1}, j}=\mathcal{F}_{s_{1}, j}^{(0)} \sigma^{s_{1}}=\bar{\Gamma}_{0}^{\left(s_{1}+1, j\right)} \sigma^{s_{1}}$ so that

$$
Q_{s_{1}, j}=\sigma^{s_{1}} \sum_{\theta \in \mathcal{T}_{s_{1}+1,0, \Gamma, j}} \operatorname{Val}(\theta) .
$$

Hence the summands in the second and third lines in (5.10) can be imagined as "some" of the trees in $\mathcal{T}_{s_{1}+1,0, \Gamma, j}$ where "some" leaves are substituted by graph elements with $h_{\ell}=\beta_{0}$. More precisely we shall consider only trees $\theta$ of the form depicted in Figure 5 , with $s_{1}+1$ nodes, $s_{0}=s_{0,0}+\ldots+s_{0, i_{0}}$ leaves, where $s_{0, \mathfrak{a}}$ is the number of the leaves with leaf label $\mathfrak{a}$, and $s_{0}^{\prime}$ graph elements with $h_{\ell}=\beta_{0}$, such that

$$
\begin{gathered}
s_{1} \mathfrak{p}+\sum_{i=0}^{i_{0}} s_{0, i} \mathfrak{h}_{i}+s_{0}^{\prime} \mathfrak{h}_{i_{0}}=\mathfrak{s}_{i_{0}}+n, \\
\sum_{i=1}^{s_{0}^{\prime}} k_{i}=\left(s_{0}^{\prime}-1\right) \mathfrak{h}_{i_{0}}+k-n,
\end{gathered}
$$

for a suitable $0 \leq n \leq k-\mathfrak{h}_{i_{0}}$, with the constraint that when $n=0$ one has $s_{0}^{\prime} \geq 2$. We shall call $\ell_{i}$ the $s_{0}^{\prime}$ lines with $h_{\ell_{i}}=\beta_{0}$. Such conditions express the condition on the sums in the second and third lines in (5.10).

The propagators of the lines exiting any among the $s_{1}+1$ nodes and the node factors of the nodes (except the root line and the node which the root line exits) are $g_{\ell}^{*}=g_{\ell}$ and $\mathcal{N}_{\mathfrak{v}}^{*}=\sigma^{b_{\mathfrak{v}}} \mathcal{N}_{\mathfrak{v}}$ with the component labels assuming the values $\widetilde{\beta}, B$, which have the rôle of $\alpha, A$, respectively. We associate with the root line a propagator

$$
g_{\ell_{0}}^{*}=-\frac{1}{C}
$$

where $C$ is defined in (5.11), while the node $\mathfrak{v}=\mathfrak{v}_{0}$ which the root line exits will have a node factor $\mathcal{N}_{\mathfrak{v}_{0}}^{*}=\mathcal{N}_{\mathfrak{v}_{0}}$ as in (6.3) Finally we associate with each leaf $\mathfrak{e}$ a leaf factor $\mathcal{N}_{\mathfrak{e}}^{*}=c_{\mathfrak{a}_{\mathfrak{e}}}$. 


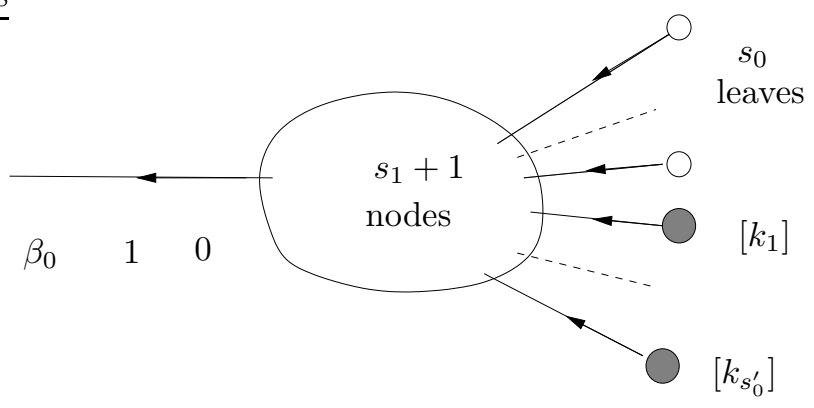

Figure 5: A tree contributing to $\beta_{0}^{[k]}$.

We now iterate such a process until only nodes or leaves appear. We shall call allowed trees all the trees obtained in such a recursive way, and we shall denote with $\Theta_{k, \nu, h}$ the set of allowed trees with order $k$, total momentum $\nu$ and total component label $h$.

Given an allowed tree $\theta$ we denote with $N(\theta), L(\theta)$ and $E(\theta)$ the set of nodes, lines and leaves of $\theta$ respectively, and we denote with $E_{\mathfrak{a}}(\theta)$ the set of leaves in $\theta$ with leaf label $\mathfrak{a}$. We point out that $E(\theta)=E_{0}(\theta) \amalg \ldots \amalg E_{i_{0}}(\theta)$. We shall define the value of $\theta$ as

$$
\operatorname{Val}^{*}(\theta)=\left(\prod_{\ell \in L(\theta)} g_{\ell}^{*}\right)\left(\prod_{\mathfrak{v} \in N(\theta)} \mathcal{N}_{\mathfrak{v}}^{*}\right)\left(\prod_{\mathfrak{c} \in E(\theta)} \mathcal{N}_{\mathfrak{c}}^{*}\right) .
$$

Finally, we denote with $\Lambda(\theta)$ the set of the lines (exiting a node) in $\theta$ with component label $h=\beta_{0}$ and with $N^{*}(\theta)$ the nodes with $b_{\mathfrak{v}}=1$; then we associate with each node in $N^{*}(\theta)$, with each leaf in $E_{\mathfrak{a}}(\theta)$ and with each line in $\Lambda(\theta)$ a weight $\mathfrak{p}, \mathfrak{h}_{\mathfrak{a}}$ and $\mathfrak{h}_{i_{0}}-\mathfrak{p}-\mathfrak{s}_{i_{0}}$, respectively, and we call order of $\theta$ the number

$$
k(\theta)=\mathfrak{p}\left|N^{*}(\theta)\right|+\left(\mathfrak{h}_{i_{0}}-\mathfrak{p}-\mathfrak{s}_{i_{0}}\right)|\Lambda(\theta)|+\sum_{\mathfrak{a}=0}^{i_{0}} \mathfrak{h}_{\mathfrak{a}}\left|E_{\mathfrak{a}}(\theta)\right| .
$$

Note that $\mathfrak{h}_{i_{0}}-\mathfrak{p}-\mathfrak{s}_{i_{0}}<0$.

Lemma 16. The Fourier coefficients $\beta_{0}^{[k]}, \widetilde{\beta}_{\nu}^{[k]}$ and $B_{\nu}^{[k]}$ can be written in terms of trees as

$$
\begin{aligned}
\beta_{0}^{[k]}=\sum_{\theta \in \Theta_{k, 0, \beta_{0}}} \operatorname{Val}^{*}(\theta), & k \geq \mathfrak{h}_{i_{0}}+1, \\
\widetilde{\beta}_{\nu}^{[k]}=\sum_{\theta \in \Theta_{k, \nu, \tilde{\beta}}} \operatorname{Val}^{*}(\theta), & k \geq \mathfrak{p}, \\
B_{\nu}^{[k]}=\sum_{\theta \in \Theta_{k, \nu, B}} \operatorname{Val}^{*}(\theta), & k \geq \mathfrak{p} .
\end{aligned}
$$

Proof. We only have to prove that an allowed tree contributing to the Fourier coefficients $\beta_{0}^{[k]}, \widetilde{\beta}_{\nu}^{[k]}$ and $B_{\nu}^{[k]}$ has order $k$. We shall perform the proof by induction on $k \geq \mathfrak{h}_{i_{0}}+1$ for the coefficients $\beta_{0}^{[k]}$, and $k \geq \mathfrak{p}$ for $\widetilde{\beta}_{\nu}^{[k]}$ and $B_{\nu}^{[k]}$. Let us set $f_{\widetilde{\beta}}=\widetilde{\beta}$ and $f_{B}=B$. An allowed tree $\theta$ contributing to $f_{h, \nu}^{[\mathfrak{p}]}$ has only one node so that $k(\theta)=\mathfrak{p}$, while an allowed tree $\bar{\theta}$ contributing to $\beta_{0}^{\left[\mathfrak{h}_{i}+1\right]}, i=0, \ldots, i_{0}$ has $s_{1}+1$ nodes, $s_{0}=s_{0,0}+\ldots+s_{0, i_{0}}$ leaves, and one line $\Lambda(\bar{\theta})$, and via the conditions (6.5) we have $s_{1} \mathfrak{p}+s_{0,0} \mathfrak{h}_{0}+\ldots+s_{0, i_{0}} \mathfrak{h}_{i_{0}}=\mathfrak{s}_{i_{0}}+1$, so that $k(\bar{\theta})=\mathfrak{h}_{i_{0}}+1$. 
Let us suppose first that for all $k^{\prime}<k$, an allowed tree $\theta^{\prime}$ contributing to $\beta_{0}^{\left[k^{\prime}\right]}$ has order $k\left(\theta^{\prime}\right)=k^{\prime}$. By the inductive hypothesis, the order of a tree $\theta$ contributing to $\beta_{0}^{[k]}$ is (we refer again to Figure 5 for notations)

$$
k(\theta)=\left(s_{1}+1\right) \mathfrak{p}+\sum_{i=0}^{i_{0}} s_{0, i} \mathfrak{h}_{i}+\sum_{i=1}^{s_{0}^{\prime}} k_{i}+\mathfrak{h}_{i_{0}}-\mathfrak{p}-\mathfrak{s}_{i_{0}},
$$

and via the conditions in (6.5) we obtain $k(\theta)=k$.

Let us suppose now that the inductive hypothesis holds for all trees $\theta^{\prime}$ contributing to $f_{h, \nu}^{\left[k^{\prime}\right]}, k^{\prime}<k$. An allowed tree $\theta$ contributing to $f_{h, \nu}^{[k]}$ is of the form depicted in Figure [6. where $s_{0, \mathfrak{a}}$ is the number of the lines exiting a leaf with leaf label $\mathfrak{a}$ and entering $\mathfrak{v}_{0}, s_{1}$ is the number of the lines exiting a node and entering $\mathfrak{v}_{0}$, and $s_{0}^{\prime}, s_{1}^{\prime}$ are the graph elements entering $\mathfrak{v}_{0}$ with component label $\beta_{0}$ and either $\widetilde{\beta}$ or $B$, respectively.

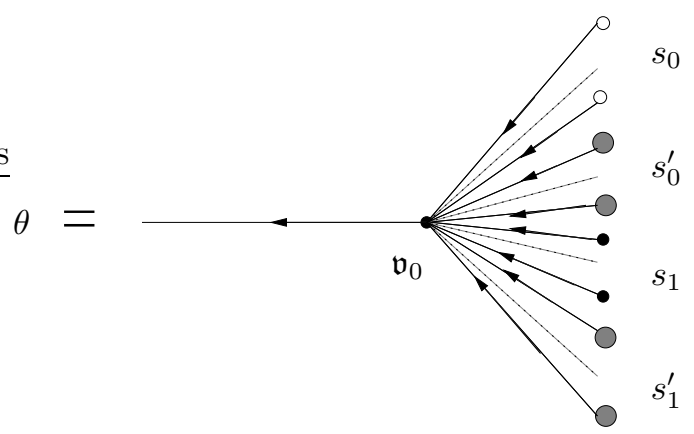

Figure 6: An allowed tree contributing to $f_{h, \nu}^{[\kappa]}$.

If $b_{\mathfrak{v}_{0}}=1$, by the inductive hypothesis the order of such a tree is given by

$$
k(\theta)=\left(s_{1}+1\right) \mathfrak{p}+\sum_{i=0}^{i_{0}} s_{0, i} \mathfrak{h}_{i}+\sum_{i=1}^{s_{0}^{\prime}+s_{1}^{\prime}} k_{i},
$$

and by the first condition in (6.2) we have $k(\theta)=k$. Otherwise if $b_{\mathfrak{v}_{0}}=0$, we have $s_{0}+s_{0}^{\prime}=0$ and, by the inductive hypothesis,

$$
k(\theta)=s_{1} \mathfrak{p}+\sum_{i=1}^{s_{1}^{\prime}} k_{i}=k
$$

via the second condition in (6.2).

Lemma 17. Let $\mathfrak{q}:=\min \left\{\mathfrak{h}_{0}, \mathfrak{p}\right\}$ and let us define

$$
M=2 \frac{\mathfrak{s}_{i_{0}}}{\mathfrak{q}}+3 .
$$

Then for all $\theta \in \Theta_{k, \nu, h}$ one has

$$
|L(\theta)| \leq M k .
$$

As the proof is rather technical we shall perform it in Appendix B

The convergence of the series (5.1) for small $\eta$ follows from the following result. 
Proposition 3. The formal solution (5.1) of the system (2.9), given by the recursive equations (5.2) and (5.12), converges for $\eta$ small enough.

Proof. By Lemma 17, the number of unlabeled trees of order $k$ is bounded by $4^{M k}$. Thus, the sum over all labels except the mode labels and the momenta is bounded by a constant to the power $k$ because all such labels can assume only a finite number of values. The bound for each node factor is the same as in Proposition 1 while the propagators can be bounded by

$$
\left|g_{\ell}^{*}\right| \leq \max \left\{\left|\frac{\omega^{\prime}\left(A_{0}\right)}{\omega^{2}}\right|,\left|\frac{1}{\omega^{\prime}\left(A_{0}\right)}\right|,\left|\frac{1}{\omega}\right|,\left|\frac{1}{C}\right|, 1\right\},
$$

so that the product over all the lines can be bounded again by a constant to the power $k$. The product over the leaves factors is again bounded by a constant to the power $k$, while the sum over the mode labels which uniquely determine the momenta can be performed by using for each node half the exponential decay factor provided by (4.10). Thus we obtain

$$
\left|\widetilde{\beta}_{\nu}^{[k]}\right| \leq C_{1} C_{2}^{k} e^{-\kappa|\nu| / 2}, \quad\left|B_{\nu}^{[k]}\right| \leq C_{1} C_{2}^{k} e^{-\kappa|\nu| / 2},
$$

for suitable constants $C_{1}$ and $C_{2}$. Hence we obtain the convergence for the series (5.1), for $|\eta| \leq C_{2}^{-1}$.

The discussion above ends the proof of Theorem 2 .

\section{Higher order subharmonic Melnikov functions}

Now we shall see how to extend the results above when the Melnikov function vanishes identically.

We are searching for a solution of the form $(\alpha(t), A(t))$ with $\alpha(t)=\alpha_{0}(t)+\beta_{0}+\widetilde{\beta}(t)$ and $A(t)=$ $A_{0}+B(t)$, where

$$
\widetilde{\beta}(t)=\sum_{\substack{\nu \in \mathbb{Z} \\ \nu \neq 0}} e^{i \nu \omega t} \beta_{\nu}\left(\varepsilon, \beta_{0}\right), \quad B(t)=\sum_{\nu \in \mathbb{Z}} e^{i \nu \omega t} B_{\nu}\left(\varepsilon, \beta_{0}\right) .
$$

First of all, we notice that we can formally write the equations of motion as

$$
\left\{\begin{array}{l}
\bar{\beta}_{\nu}^{(k)}\left(\beta_{0}\right)=\frac{\bar{\Phi}_{\nu}^{(k)}\left(\beta_{0}\right)}{i \omega \nu}+\omega^{\prime}\left(A_{0}\right) \frac{\bar{\Gamma}_{\nu}^{(k)}\left(\beta_{0}\right)}{(i \omega \nu)^{2}}, \quad \bar{B}_{\nu}^{(k)}\left(\beta_{0}\right)=\frac{\bar{\Gamma}_{\nu}^{(k)}\left(\beta_{0}\right)}{i \omega \nu}, \quad \nu \neq 0 \\
\bar{B}_{0}^{(k)}\left(\beta_{0}\right)=-\frac{\bar{\Phi}_{0}^{(k)}\left(\beta_{0}\right)}{\omega^{\prime}\left(A_{0}\right)} .
\end{array}\right.
$$

where the notations in (2.22) have been used, up to any order $k$, provided

$$
\bar{\Gamma}_{0}\left(\varepsilon, \beta_{0}\right)=0 .
$$

If $M\left(t_{0}\right)$ vanishes identically, by (2.24) we have $\bar{\Gamma}_{0}^{(1, j)}=0$ for all $j \geq 0$, that is $\bar{\Gamma}_{0}^{(1)}\left(\beta_{0}\right)=0$, for all $\beta_{0}$, and hence $\bar{\Gamma}_{0}\left(\varepsilon, \beta_{0}\right)=\varepsilon^{2} \mathcal{F}^{(2)}\left(\varepsilon, \beta_{0}\right)$, with $\mathcal{F}^{(2)}$ a suitable function analytic in $\varepsilon, \beta_{0}$.

Thus, we can solve the equations of motion up to the first order in $\varepsilon$, and the parameter $\beta_{0}$ is left undetermined. More precisely we obtain

$$
\beta_{\nu}=\varepsilon \bar{\beta}_{\nu}^{(1)}+\varepsilon \widetilde{\beta}_{\nu}^{(1)}\left(\varepsilon, \beta_{0}\right), \quad B_{\nu}=\varepsilon \bar{B}_{\nu}^{(1)}+\varepsilon \widetilde{B}_{\nu}^{(1)}\left(\varepsilon, \beta_{0}\right),
$$

where $\bar{\beta}_{\nu}^{(1)}, \bar{B}_{\nu}^{(1)}$ solve the equation of motion up to the first order in $\varepsilon$, while $\widetilde{\beta}_{\nu}^{(1)}, \widetilde{B}_{\nu}^{(1)}$ are the corrections to be determined. 
Now, let us set

$$
M_{0}\left(t_{0}\right)=M\left(t_{0}\right), \quad M_{1}\left(t_{0}\right)=\bar{\Gamma}_{0}^{(2)}\left(0, t_{0}\right),
$$

where $\bar{\Gamma}_{\nu}^{(k)}\left(\beta_{0}, t_{0}\right)=\bar{\Gamma}_{\nu}^{(k)}\left(\beta_{0}\right)$, i.e. we are stressing the dependence of $\bar{\Gamma}_{\nu}^{(k, j)}$ on $t_{0}$. We refer to $M_{1}\left(t_{0}\right)$ as the second order subharmonic Melnikov function. Notice that $M_{0}\left(t_{0}\right)=\bar{\Gamma}_{0}^{(1)}\left(0, t_{0}\right)$.

If there exist $t_{0} \in[0,2 \pi)$ and $\mathfrak{n}_{1} \in \mathbb{N}$ such that $t_{0}$ is a zero of order $\mathfrak{n}_{1}$ for the second order subharmonic Melnikov function, that is

$$
\frac{\mathrm{d}^{k}}{\mathrm{~d} t_{0}^{k}} M_{1}\left(t_{0}\right)=0 \quad \forall 0 \leq k \leq \mathfrak{n}_{1}-1, \quad D=D\left(t_{0}\right):=\frac{\mathrm{d}^{\mathfrak{n}_{1}}}{\mathrm{~d} t_{0}^{\mathfrak{n}_{1}}} M_{1}\left(t_{0}\right) \neq 0,
$$

then we can repeat the analysis of the previous Sections to obtain the existence of a subharmonic solution. In fact, we have

$$
\mathcal{F}^{(2)}\left(\varepsilon, \beta_{0}\right):=\sum_{k, j \geq 0} \varepsilon^{k} \beta_{0}^{j} \mathcal{F}_{k, j}^{(2)}, \quad \mathcal{F}_{k, j}^{(2)}=\bar{\Gamma}_{0}^{(k+2, j)}\left(t_{0}\right),
$$

where $t_{0}$ has to be fixed as the zero of $M_{1}\left(t_{0}\right)$, so that, as

$$
\left(-\omega\left(A_{0}\right)\right)^{-j} \frac{\mathrm{d}^{j}}{\mathrm{~d} t_{0}^{j}} M_{1}\left(t_{0}\right)=j ! \bar{\Gamma}_{0}^{(2, j)}\left(t_{0}\right)
$$

for all $j$, as proved in [6] with a different notation, we can construct the Newton polygon of $\mathcal{F}^{(2)}$, which is $\beta_{0}$-general of order $\mathfrak{n}_{1}$ by (7.6), to obtain $\widetilde{\beta}^{(1)}, \widetilde{B}^{(1)}$ and $\beta_{0}$ as Puiseux series in $\varepsilon$, provided at each step of the iteration of the Newton-Puiseux algorithm one has a real root.

Otherwise, if $M_{1}\left(t_{0}\right)$ vanishes identically, we have $\bar{\Gamma}_{0}^{(2)}\left(\beta_{0}\right)=0$ for all $\beta_{0}$, so that we can solve the equations of motion up to the second order in $\varepsilon$ and the parameter $\beta_{0}$ is still undetermined. Hence we set $M_{2}\left(t_{0}\right)=\bar{\Gamma}_{0}^{(3)}\left(0, t_{0}\right)$ and so on.

In general if $M_{k^{\prime}}\left(t_{0}\right) \equiv 0$, for all $k^{\prime}=0, \ldots, \kappa-1$, we have $\bar{\Gamma}_{0}\left(\varepsilon, \beta_{0}\right)=\varepsilon^{k^{\prime}} \mathcal{F}^{\left(k^{\prime}\right)}\left(\varepsilon, \beta_{0}\right)$, so that we can solve the equations of motion up to the $\kappa$-th order in $\varepsilon$, and obtain

$$
\begin{aligned}
& \beta_{\nu}=\varepsilon \bar{\beta}_{\nu}^{(1)}+\ldots+\varepsilon^{\kappa} \bar{\beta}_{\nu}^{(\kappa)}+\varepsilon^{\kappa} \widetilde{\beta}_{\nu}^{(k)}\left(\varepsilon, \beta_{0}\right), \\
& B_{\nu}=\varepsilon \bar{B}_{\nu}^{(1)}+\ldots+\varepsilon^{\kappa} \bar{B}_{\nu}^{(\kappa)}+\varepsilon^{\kappa} \widetilde{B}_{\nu}^{(k)}\left(\varepsilon, \beta_{0}\right),
\end{aligned}
$$

where $\bar{\beta}_{\nu}^{\left(k^{\prime}\right)}, \bar{B}_{\nu}^{\left(k^{\prime}\right)}, k^{\prime}=0, \ldots, \kappa-1$ solve the equation of motion up to the $\kappa$-th order in $\varepsilon$, while $\widetilde{\beta}_{\nu}^{(\kappa)}$, $\widetilde{B}_{\nu}^{(\kappa)}$ are the correction to be determined.

Hence we can weaken Hypotheses 2 and 3 as follows.

Hypothesis 4. There exists $\kappa \geq 0$ such that for all $k^{\prime}=0, \ldots, \kappa-1, M_{k^{\prime}}\left(t_{0}\right)$ vanishes identically, and there exist $t_{0} \in[0,2 \pi)$ and $\mathfrak{n} \in \mathbb{N}$ such that

$$
\frac{\mathrm{d}^{j}}{\mathrm{~d} t_{0}^{j}} M_{\kappa}\left(t_{0}\right)=0 \quad \forall 0 \leq j \leq \mathfrak{n}-1, \quad D=D\left(t_{0}\right):=\frac{\mathrm{d}^{\mathfrak{n}}}{\mathrm{d} t_{0}^{\mathfrak{n}}} M_{\kappa}\left(t_{0}\right) \neq 0,
$$

that is $t_{0}$ is a zero of order $\mathfrak{n}$ for the $\kappa$-th order subharmonic Melnikov function.

Hypothesis 5. There exists $i_{0} \geq 0$ such that at the $i_{0}$-th step of the iteration of the Newton-Puiseux algorithm for $\mathcal{F}^{(\kappa)}$, there exists a polynomial $P^{\left(i_{0}\right)}=P^{\left(i_{0}\right)}(c)$ which has a simple root $c^{*} \in \mathbb{R}$.

Thus we have the following result. 
Theorem 3. Consider a periodic solution with frequency $\omega=p / q$ for the system (2.1), and assume that Hypotheses 1, 4 and 5 are satisfied. Then there exists an explicitly computable value $\varepsilon_{0}>0$ such that for $|\varepsilon|<\varepsilon_{0}$ the system (2.1) has at least one subharmonic solution of order $q / p$. Such a solution admits a convergent power series in $|\varepsilon|^{1 / \mathfrak{n} !}$, and hence a convergent Puiseux series in $|\varepsilon|$.

The proof can be easily obtained suitably modifying the proof of Theorem 2 .

Now, call $\Re_{n}^{(\kappa)}$ the set of real roots of the polynomials obtained at the $n$-th step of iteration of the Newton-Puiseux process for $\mathcal{F}^{(\kappa)}$. Again if $\mathfrak{n}$ is even we can not say a priori whether a formal solution exists at all. However, if $\Re_{n}^{(\kappa)} \neq \emptyset$ for all $n \geq 0$, then we obtain a convergent Puiseux series as in Section 3 .

Finally, as a corollary, we have the following result.

Theorem 4. Consider a periodic solution with frequency $\omega=p / q$ for the system (2.1). Assume that Hypotheses 1 and 4 are satisfied with $\mathfrak{n}$ odd. Then for $\varepsilon$ small enough the system (2.1) has at least one subharmonic solution of order $q / p$. Such a solution admits a convergent power series in $|\varepsilon|^{\mathfrak{n} !}$, and hence a convergent Puiseux series in $\varepsilon$.

Again the proof is a suitable modification of the proof of Theorem 1

Acknowledgements. We thank Edoardo Sernesi for useful discussions.

\section{A On the genericity of Hypothesis 3}

Here we want to show that Hypothesis 3 is generic on the space of the coefficients of the polynomials. More precisely, we shall show that given a polynomial of the form

$$
P(a, c)=\sum_{i=0}^{n} a_{n-i} c^{i}, \quad n \geq 1, \quad a:=\left(a_{0}, \ldots, a_{n}\right),
$$

the set of parameters $\left(a_{0}, \ldots, a_{n}\right) \in \mathbb{R}^{n+1}$ for which $P(a, c)$ has multiples roots, is a proper Zariskiclosed 1 subset of $\mathbb{R}^{n+1}$. Notice that a polynomial $P=P(a, c)$ has a multiple root $c^{*}$ if and only if also the derivative $\partial P / \partial c$ vanishes at $c^{*}$.

Recall that, given two polynomials

$$
P_{1}(c)=\sum_{i=0}^{n} a_{n-i} c^{i}, \quad P_{2}(c)=\sum_{i=0}^{m} b_{m-i} c^{i},
$$

with $n, m \geq 1$, the Sylvester matrix of $P_{1}, P_{2}$ is an $n+m$ square matrix where the columns 1 to $m$ are formed by "shifted sequences" of the coefficients of $P_{1}$, while the columns $m+1$ to $m+n$ are formed by "shifted sequences" of the coefficients of $P_{2}$, i.e.

$$
\operatorname{Syl}\left(P_{1}, P_{2}\right):=\left(\begin{array}{cccccccc}
a_{0} & 0 & \ldots & 0 & b_{0} & 0 & \ldots & 0 \\
a_{1} & a_{0} & \ldots & 0 & b_{1} & b_{0} & \ldots & 0 \\
\vdots & \vdots & \ddots & \vdots & \vdots & \vdots & \ddots & \vdots \\
0 & 0 & \ldots & a_{n-1} & 0 & 0 & \ldots & b_{m-1} \\
0 & 0 & \ldots & a_{n} & 0 & 0 & \ldots & b_{m}
\end{array}\right),
$$

and the resultant $R\left(P_{1}, P_{2}\right)$ of $P_{1}, P_{2}$ is defined as the determinant of the Sylvester matrix.

\footnotetext{
${ }^{1}$ See for instance [12.
} 
Lemma 18. Let $c_{1,1}, \ldots, c_{1, n}$ and $c_{2,1}, \ldots, c_{2, m}$ be the complex roots of $P_{1}, P_{2}$ respectively. Then

$$
R\left(P_{1}, P_{2}\right)=a_{0}^{m} b_{0}^{n} \prod_{i=1}^{n} \prod_{j=1}^{m}\left(c_{1, i}-c_{2, j}\right) .
$$

A complete proof is performed for instance in [13. In particular, Lemma 18 implies that two polynomials have a common root if and only if $R\left(P_{1}, P_{2}\right)=0$.

Recall also that given a polynomial $P=P(c)$, the discriminant $D(P)$ of $P$ is the resultant of $P$ and its first derivative with respect to $c$, i.e. $D(P):=R\left(P, P^{\prime}\right)$, where $P^{\prime}:=\mathrm{d} P / \mathrm{d} c$. Thus, a polynomial $P=P(a, c)$ of the form (A.1) has a multiple root if and only if its discriminant is equal to zero.

Now let us consider the set

$$
V:=\left\{a=\left(a_{0}, \ldots, a_{n}\right) \in \mathbb{R}^{n+1}: P(a, c) \text { has a multiple root }\right\} .
$$

The discriminant of $P(a, c)$ is a polynomial in the parameters $a=\left(a_{0}, \ldots, a_{n}\right)$ i.e. $D_{P}(a)=D(P) \in$ $\mathbb{R}\left[a_{0}, \ldots, a_{n}\right]$, hence we can write

$$
V=\left\{a=\left(a_{0}, \ldots, a_{n}\right) \in \mathbb{R}^{n+1}: D_{P}(a)=0\right\} .
$$

Such a set is, by definition, a proper Zariski-closed subset of $\mathbb{R}^{n+1}$.

As the complement of a proper Zariski-closed subset of $\mathbb{R}^{n+1}$ is open and dense also in the Euclidean topology, then Hypothesis 3 is generic.

\section{B Proof of Lemma 17}

First we shall prove by induction on $k$ that for all $\theta \in \Theta_{k, 0, \beta_{0}}$ one has

$$
|L(\theta)| \leq M\left(k-\mathfrak{h}_{i_{0}}\right)-\left(1+\frac{\mathfrak{s}_{i_{0}}}{\mathfrak{q}}\right)
$$

for all $k \geq \mathfrak{h}_{i_{0}}+1$.

For $k=\mathfrak{h}_{i_{0}}+1$ one has

$$
\beta_{0}^{\left[\mathfrak{h}_{i_{0}}+1\right]}=-\frac{1}{C} \sum_{\substack{m_{1}, j \geq 0 \\ m_{0}+\ldots+m_{i_{0}}=j \\ s_{1} \mathfrak{p}+m_{0} \mathfrak{h}_{0}+\ldots+m_{i_{0}} \mathfrak{h}_{i_{0}}=\mathfrak{s}_{i_{0}}+1}} J\left(j, m_{0}, \ldots, m_{i_{0}}, m\right) Q_{s_{1}, j} c_{0}^{m_{0}} \ldots c_{i_{0}}^{m_{i_{0}}},
$$

so that any tree $\theta$ contributing to $\beta_{0}^{\left[\mathfrak{h}_{i}+1\right]}$ has $s_{1}+1$ nodes and $j$ leaves, hence $|L(\theta)|=s_{1}+1+j$.

Notice that $\mathfrak{q} \leq \mathfrak{h}_{0} \leq \mathfrak{h}_{1} \leq \ldots \leq \mathfrak{h}_{i_{0}}$, hence one has

$$
|L(\theta)|=1+s_{1}+j \leq 1+\frac{\mathfrak{s}_{i_{0}}+1}{\mathfrak{q}} .
$$

Moreover for $k=\mathfrak{h}_{i_{0}}+1$ the r.h.s. in (B.1) is equal to $2+\mathfrak{s}_{i_{0}} / \mathfrak{q}$, so that the bound (B.1) holds, because one has $\mathfrak{q} \geq 1$. Assume now that the bound (B.1) holds for all $k^{\prime}<k$ and let us show that then it holds also for $k$.

We call $M_{0}=M \mathfrak{h}_{i_{0}}+1+\mathfrak{s}_{i_{0}} / \mathfrak{q}$, so that the inductive hypothesis can be written as

$$
\left|L\left(\theta^{\prime}\right)\right| \leq M k\left(\theta^{\prime}\right)-M_{0},
$$


for all $\theta^{\prime} \in \Theta_{k^{\prime}, 0, \beta_{0}}, k^{\prime}<k$.

By the inductive hypothesis, we have (cf. Figure 5)

$$
|L(\theta)| \leq 1+s_{1}+s_{0}-s_{0}^{\prime} M_{0}+M \sum_{i=1}^{s_{0}^{\prime}} k\left(\theta_{i}\right)
$$

for suitable $\theta_{1}, \ldots, \theta_{s_{0}^{\prime}}$ depending on $\theta$.

Let us set $m:=k-\mathfrak{h}_{i_{0}} \geq 1$. Hence, via the conditions (6.5) we can write (B.5) as

$$
|L(\theta)| \leq 1+s_{1}+s_{0}-s_{0}^{\prime} M_{0}+M\left(\mathfrak{s}_{i_{0}}+m-s_{1} \mathfrak{p}-\sum_{i=0}^{i_{0}} s_{0, i} \mathfrak{h}_{i}\right) .
$$

Hence we shall prove that

$$
1+s_{1}+s_{0}-s_{0}^{\prime} M_{0}+M\left(\mathfrak{s}_{i_{0}}+m-s_{1} \mathfrak{p}-\sum_{i=0}^{i_{0}} s_{0, i} \mathfrak{h}_{i}\right) \leq m M-1-\frac{\mathfrak{s}_{i_{0}}}{\mathfrak{q}},
$$

or, in other words

$$
\left(s_{1} \mathfrak{p}+\sum_{i=0}^{i_{0}} s_{0, i} \mathfrak{h}_{i}\right) M+s_{0}^{\prime} M_{0} \geq \mathfrak{s}_{i_{0}} M+s_{0}+s_{1}+\frac{\mathfrak{s}_{i_{0}}}{\mathfrak{q}}+2,
$$

for all $s_{0}, s_{0}^{\prime}, s_{1} \geq 0$ admitted by conditions (6.5).

First of all for $s_{0}^{\prime}=0$ by the first condition in (6.5) we have $s_{1} \mathfrak{p}+s_{0,0} \mathfrak{h}_{0}+\ldots+s_{0, i_{0}} \mathfrak{h}_{i_{0}}=\mathfrak{s}_{i_{0}}+m$. Moreover $\left(s_{1}+s_{0}\right) \mathfrak{q} \leq s_{1} \mathfrak{p}+s_{0, i_{0}} \mathfrak{h}_{0}+\ldots+s_{0, i_{0}} \mathfrak{h}_{i_{0}}=\mathfrak{s}_{i_{0}}+m$, hence

$$
s_{1}+s_{0} \leq \frac{\mathfrak{s}_{i_{0}}+m}{\mathfrak{q}},
$$

so that one obtain (B.8) if

$$
m M \geq 2 \frac{\mathfrak{s}_{i_{0}}}{\mathfrak{q}}+2+\frac{m}{\mathfrak{q}}
$$

hence one needs

$$
m\left(2 \frac{\mathfrak{s}_{i_{0}}}{\mathfrak{q}}+3\right) \geq 2 \frac{\mathfrak{s}_{i_{0}}}{\mathfrak{q}}+2+\frac{m}{\mathfrak{q}}
$$

that is satisfied for all $m \geq 1$.

For $s_{0}^{\prime}=1$ the first conditions (6.5) can be written as $s_{1} \mathfrak{p}+s_{0,0} \mathfrak{h}_{0}+\ldots+\left(s_{0, i_{0}}+1\right) \mathfrak{h}_{i_{0}}=\mathfrak{s}_{i_{0}}+n$, so that

$$
s_{1}+s_{0} \leq \frac{\mathfrak{s}_{i_{0}}-\mathfrak{h}_{i_{0}}+n}{\mathfrak{q}} .
$$

Hence we obtain (B.8) if

$$
n M \geq \frac{\mathfrak{s}_{i_{0}}+n-\mathfrak{h}_{i_{0}}}{\mathfrak{q}}+1,
$$

and again (B.13) is satisfied because $n=k-k_{1} \geq 1$.

Finally for $s_{0}^{\prime} \geq 2$ the first condition in (6.5) can be written $s_{1} \mathfrak{p}+s_{0,0} \mathfrak{h}_{0}+\ldots+\left(s_{0, i_{0}}+s_{0}^{\prime}\right) \mathfrak{h}_{i_{0}} \geq \mathfrak{s}_{i_{0}}$, so that $s_{1}+s_{0}<s_{1}+s_{0}+s_{0}^{\prime} \leq \frac{\mathfrak{s}_{0}}{\mathfrak{q}}$, and we obtain (B.8) by requiring

$$
\mathfrak{s}_{i_{0}} M+s_{0}^{\prime}\left(\frac{\mathfrak{s}_{i_{0}}}{\mathfrak{q}}+1\right) \geq \mathfrak{s}_{i_{0}} M+2 \frac{\mathfrak{s}_{i_{0}}}{\mathfrak{q}}+2
$$


that is satisfied as we are assuming $s_{0}^{\prime} \geq 2$.

This exhausts the discussion over all the choices of $s_{0}, s_{0}^{\prime}, s_{1}$.

Let us show now that

$$
|L(\theta)| \leq M k-1,
$$

for all $\theta \in \Theta_{k, \nu, f}, f=\widetilde{\beta}, B, k \geq \mathfrak{p}$.

Again recall that a tree $\theta \in \Theta_{k, \nu, f}$ contributes to $f_{\nu}^{[k]}$ with $f=\widetilde{\beta}, B$, so that the bound (B.15) is trivially satisfied for $k=\mathfrak{p}$ because one has $|L(\theta)|=1$.

Let us suppose now that the bound holds for all $\mathfrak{p}<k^{\prime}<k$; again we shall prove that then it holds also for $k$.

Recall that a tree contributing to $f_{\nu}^{[k]}$ is of the form depicted in Figure [6, where $s_{0, \mathfrak{a}}$ is the number of the lines exiting a leaf with leaf label $\mathfrak{a}$ and entering $\mathfrak{v}_{0}, s_{0}=s_{0,0}+\ldots+s_{0, i_{0}}, s_{1}$ is the number of the lines exiting a node and entering $\mathfrak{v}_{0}$, and $s_{0}^{\prime}, s_{1}^{\prime}$ are the graph elements entering $\mathfrak{v}_{0}$ with component label $\beta_{0}$ and $f$ respectively. Hence, by the inductive hypothesis and by the bound (B.1), we have

$$
|L(\theta)| \leq 1+s_{0}+s_{1}-s_{0}^{\prime} M_{0}-s_{1}^{\prime}+M \sum_{i=1}^{s_{0}^{\prime}+s_{1}^{\prime}} k\left(\theta_{i}\right)
$$

for suitable $\theta_{1}, \ldots, \theta_{s_{0}^{\prime}}$ depending on $\theta$.

Let us supposte first $b_{\mathfrak{v}_{0}}=1$; thus, via the first condition in (6.2), we have to prove the bound

$$
1+s_{0}+s_{1}+M\left(k-\mathfrak{p}-s_{0,0} \mathfrak{h}_{0}-\ldots-s_{0, i_{0}} \mathfrak{h}_{i_{0}}-s_{1} \mathfrak{p}\right)-s_{0}^{\prime} M_{0}-s_{1}^{\prime} \leq M k-1,
$$

or, in other words,

$$
\sum_{i=0}^{i_{0}} s_{0, i}\left(M \mathfrak{h}_{i}-1\right)+s_{1}(M \mathfrak{p}-1)+M \mathfrak{p}+s_{0}^{\prime} M_{0}+s_{1}^{\prime} \geq 2,
$$

and this is obviously satisfied as $M \mathfrak{h}_{i}, M \mathfrak{p} \geq 3$.

Finally if $b_{\mathfrak{v}_{0}}=0$ we have

$$
\sum_{i=1}^{s_{1}^{\prime}} k\left(\theta_{i}\right)=k-s_{1} \mathfrak{p}, \quad s_{0,0}+\ldots+s_{0, i_{0}}+s_{0}^{\prime}=0, \quad s_{1}+s_{1}^{\prime} \geq 2,
$$

so that, by the second condition in (6.2), we have to prove the bound

$$
1+s_{1}+M\left(k-s_{1} \mathfrak{p}\right)-s_{1}^{\prime} \leq M k-1,
$$

or, in other words $s_{1}(M \mathfrak{p}-1)+s_{1}^{\prime} \geq 2$, and again this is obviously satisfied as $s_{1}+s_{1}^{\prime} \geq 2$ and $M \mathfrak{p}>1$.

\section{References}

[1] A.A. Andronov, E.A. Leontovich, I.I. Gordon, A.G. Maŭer, Theory of bifurcations of dynamic systems on a plane, Halsted Press, Israel Program for Scientific Translations, Jerusalem-London, 1973.

[2] E. Brieskorn, H. Knörrer, Plane algebraic curves, Birkhäuser, Basel, 1986.

[3] S.-N. Chow, J.K. Hale, Methods of bifurcation theory, Grundlehren der Mathematischen Wissenschaften 251, Springer-Verlag, New York-Berlin, 1982. 
[4] G. Gallavotti, G. Gentile, A. Giuliani, Fractional Lindstedt series, J. Math. Phys. 47 (2006), no. 1, 012702, $33 \mathrm{pp}$.

[5] G. Gentile, M. Bartuccelli, J. Deane, Summation of divergent series and Borel summability for strongly dissipative differential equations with periodic or quasiperiodic forcing terms, J. Math. Phys. 46 (2005), no. 6, 062704, $20 \mathrm{pp}$.

[6] G. Gentile, M. Bartuccelli, J. Deane, Bifurcation curves of subharmonic solutions and Melnikov theory under degeneracies, Rev. Math. Phys. 19 (2007), no. 3, 307-348.

[7] J. Guckenheimer, Ph. Holmes, Nonlinear oscillations, dynamical systems, and bifurcations of vector fields, Applied Mathematical Sciences 42, Springer-Verlag, New York, 1990.

[8] V.K. Melnikov, On the stability of a center for time-periodic perturbations, Trudy Moskov. Mat. Obšč. 12 (1963), 3-52; translated in Trans. Moscow Math. Soc. 12 (1963), 1-57.

[9] L.M. Perko, Global families of limit cycles of planar analytic systems, Trans. Amer. Math. Soc. 322 (1990), no. $2,627-656$.

[10] L.M. Perko, Bifurcation of limit cycles: geometric theory, Proc. Amer. Math. Soc. 114 (1992), no. 1, 225-236.

[11] M.V. Puiseux, Recherches sur les fonctions algébriques, J. Math. Pures Appl. 15 (1850), 365-480.

[12] I. Shafarevich, Basic algebraic geometry vol. 1, Springer-Verlag, Berlin, 1977.

[13] B.L. van der Waerden, Algebra. Vol. 1, Springer-Verlag, New York, 1991.

[14] Zh.F. Zhang, B.Y. Li, High order Melnikov functions and the problem of uniformity in global bifurcation, Ann. Mat. Pura Appl. (4) 161 (1992), 181-212. 